\title{
Genetic variants associated with Alzheimer's disease confer different cerebral cortex cell-type population structure
}

Zeran Li', Jorge L. Del-Aguila1, Umber Dube ${ }^{1,2}$, John Budde, Rita Martinez ${ }^{1}$, Kathleen Black, Qingli Xiao ${ }^{3}$, Nigel J. Cairns 3,4,5, The Dominantly Inherited Alzheimer Network (DIAN), Joseph D. Dougherty 1,7, Jin-Moo Lee ${ }^{3}$, John C. Morris ${ }^{3,5,6}$, Randall J. Bateman ${ }^{3,5,6}$, Celeste M. Karch ${ }^{1}$, Carlos Cruchaga ${ }^{1,5,6^{*}}$ and Oscar Harari ${ }^{\text {* }^{*}}$

\begin{abstract}
Background: Alzheimer's disease (AD) is characterized by neuronal loss and astrocytosis in the cerebral cortex. However, the specific effects that pathological mutations and coding variants associated with AD have on the cellular composition of the brain are often ignored.

Methods: We developed and optimized a cell-type-specific expression reference panel and employed digital deconvolution methods to determine brain cellular distribution in three independent transcriptomic studies.

Results: We found that neuronal and astrocyte relative proportions differ between healthy and diseased brains and also among AD cases that carry specific genetic risk variants. Brain carriers of pathogenic mutations in APP, PSEN1, or PSEN2 presented lower neuron and higher astrocyte relative proportions compared to sporadic AD. Similarly, the APOE $\varepsilon 4$ allele also showed decreased neuronal and increased astrocyte relative proportions compared to AD noncarriers. In contrast, carriers of variants in TREM2 risk showed a lower degree of neuronal loss compared to matched AD cases in multiple independent studies.

Conclusions: These findings suggest that genetic risk factors associated with AD etiology have a specific imprinting in the cellular composition of AD brains. Our digital deconvolution reference panel provides an enhanced understanding of the fundamental molecular mechanisms underlying neurodegeneration, enabling the analysis of large bulk RNA-sequencing studies for cell composition and suggests that correcting for the cellular structure when performing transcriptomic analysis will lead to novel insights of AD.
\end{abstract}

Keywords: Digital deconvolution, Alzheimer's disease, Brain cellular composition, Bulk RNA-sequencing, Autosomal dominant AD, TREM2

\section{Background}

Alzheimer's disease (AD) is a neurodegenerative disorder characterized clinically by gradual and progressive memory loss and pathologically by the presence of senile plaques (A $\beta$ deposits) and neurofibrillary tangles (NFTs, Tau deposits) in the brain [1]. AD has a substantial but heterogeneous genetic component. Mutations in the amyloid-beta

\footnotetext{
*Correspondence: ccruchaga@wustl.edu; harario@wustl.edu

${ }^{1}$ Department of Psychiatry, Washington University School of Medicine, $660 \mathrm{~S}$. Euclid Ave. B8134, St. Louis, MO 63110, USA

Full list of author information is available at the end of the article
}

precursor protein $(A P P)$ and Presenilin genes (PSEN1 and PSEN2) $[2,3]$ cause autosomal dominant AD (ADAD) which is typically associated with early-onset ( $<65$ years). In contrast, the most common manifestation of $\mathrm{AD}$ presents late-onset (LOAD) and accounts for the majority of the cases (90-95\%). Despite appearing sporadic in nature, a complex genetic architecture underlies LOAD risk. $A P O E \& 4$ is the most common genetic risk factor, increasing the risk in three- to eightfold [4]. In addition, recent whole genome and whole exome analyses have identified rare coding variants in TREM2 [5, 6], PLD3 [7],

(c) The Author(s). 2018 Open Access This article is distributed under the terms of the Creative Commons Attribution 4.0 International License (http://creativecommons.org/licenses/by/4.0/), which permits unrestricted use, distribution, and 
$A B C A 7[8,9]$, and SORL1 $[10,11]$ that are associated with $\mathrm{AD}$ and confer risk comparable to that of carrying one $A P O E \& 4$ allele. Besides age at onset, the clinical presentations of LOAD and ADAD are remarkably similar with an amnestic and cognitive impairment phenotype [12, 13]. A minor fraction of cases of ADAD have additional neurological findings, sometimes also seen in $\operatorname{LOAD}[12,13]$.

Altered cellular composition is associated with $\mathrm{AD}$ progression and decline in cognition. Neuronal loss in the hippocampus is characteristic in the initial stages of $\mathrm{AD}$, which could explain early memory disturbances [14, 15]. As the disease progresses, neuronal death is observed throughout the cerebral cortex. Furthermore, $25 \%$ of cognitively normal individuals who die by the age of $\sim 75$ years also presented substantial cerebral lesions that resemble $\mathrm{AD}$ pathology, including amyloid plaque, NFTs, and neuronal loss [16]. Thus, the identification of the brain cellular population structure is essential for understanding neurodegenerative disease progression [17]. However, stereology protocols for counting neurons can be tedious, require extensive training, and are susceptible to technical artifacts which may lead to biased quantification of cell-type distributions [17].

Recently there has been a growing interest in understanding the transcriptomic changes attributed to $\mathrm{AD}$ [18-25], as these may point to underlying molecular mechanisms of disease. These studies are typically designed to analyze the expression profiles of large cohorts ascertained from homogenized regions of the brain (e.g. bulk RNA-sequencing [RNA-seq]) of affected and control donors. However, as bulk RNA-seq captures the gene expression of all the constituent cells in the sampled tissue; the altered cellular composition associated with $\mathrm{AD}$ has been reported to confound downstream analyses [20].

Digital deconvolution approaches enhance the interrogation of expression profiles to identify the cellular population structure of individual samples, alleviating the requirement of additional neurostereology procedures. These approaches have been developed, tested, and applied to ascertain cellular composition altered in many traits [26-29]. However, digital deconvolution has not been applied to identify the cellular population structure from RNA-seq from human brain of AD cases and controls. Technical constraints restrict the dissociation of cells from the brains for very specific conditions [30-32]. Nevertheless, a limited number of RNA-seq from isolated cell populations from the brain have been generated [30-32]. Using these resources, we are now able to generate a reference panel for digital deconvolution of human brain bulk RNA-seq data.

We sought to investigate the cellular population structure in $\mathrm{AD}$ by analyzing RNA-seq from multiple brain regions of LOAD participants. To do so, we assembled a novel brain reference panel and evaluated the accuracy of digital deconvolution methods by analyzing additional cell-type-specific RNA-seq samples and by creating synthetic admixtures with defined cellular distributions. Then we analyzed large cohorts of pathologically confirmed AD cases and controls $(n=613)$ and verified that our model predicts cellular distribution patterns consistent with neurodegeneration. Finally, we generated RNA-seq from the parietal lobe of participants from the Charles F. and Joanne Knight Alzheimer's Disease Research Center (Knight-ADRC) [33], including non-demented controls, LOAD cases, with enriched proportions of carriers of high-risk coding variants associated with $\mathrm{AD}$, and also ADAD from The Dominantly Inherited Alzheimer Network [34] (DIAN). We compared the cell composition in ADAD and LOAD; and also evaluated differences among carriers of coding high-risk variants in PLD3, TREM2, and APOE \&4. Our findings indicate that cell-type composition differs among carriers of specific genetic risk factors, which might be revealing distinct pathogenic mechanisms contributing to disease etiology.

\section{Methods \\ Subjects and samples \\ DIAN and Knight-ADRC}

Parietal lobe tissue of post-mortem brain was obtained with informed consent for research use and was approved by the review board of Washington University in St. Louis. RNA was extracted from frozen brain using Tissue Lyser LT and RNeasy Mini Kit (Qiagen, Hilden, Germany). RNA-seq paired-end reads with read lengths of $2 \times 150$ bp were generated using Illumina HiSeq 4000 with a mean coverage of 80 million reads per sample (Table 1; Additional file 1: Table S1). RNA-seq was generated for 19 brains from DIAN, 84 brains with LOAD and 16 non-demented controls from Knight-ADRC [33]. The AD brains selected from Knight-ADRC are enriched for carrier of variants in TREM2 $(\mathrm{n}=20$; Additional file 1: Table S1) and PLD3 ( $\mathrm{n}=33$; Additional file 1: Table S1). The clinical status of participants was neuropathologically confirmed [35]. We identified three additional participants from the Knight-ADRC study with PSEN1 (A79V, I143T, S170F) mutations. Clinical Dementia Rating (CDR) scores were obtained during regular visits throughout the study before the subject's decease [36]. A range of other pathological measurements were collected during autopsy including Braak staging, as previously described [37].

RNA was extracted from frozen brain tissues using Tissue Lyser LT and RNeasy Mini Kit (Qiagen, Hilden, Germany) following the manufacturer's instruction. RIN (RNA integrity) and DV200 were measured with RNA 6000 Pico Assay using Bioanalyzer 2100 (Agilent 
Table 1 Demographics and disease status of cohorts from four brain bank resources

\begin{tabular}{lllll}
\hline & Mayo & MSBB & DIAN & Knight-ADRC \\
\hline Sample size & 191 & 300 & 19 & 103 \\
Age (years) & $83 \pm 7.77$ & $83.3 \pm 7.55$ & $50.6 \pm 7.06$ & $85.1 \pm 9.78$ \\
Male (\%) & 45.5 & 36 & 68.4 & 38.8 \\
APOE ع4+ (\%) & 33.2 & 31.7 & 14.3 & 45.6 \\
Brain weight & - & - & $1187.7 \pm 184.5$ & $1138.1 \pm 142.5$ \\
AD & 82 & 135 & 19 & 87 \\
PA & 29 & 0 & 0 & 0 \\
Control & 80 & 85 & 0 & 16 \\
$C D R=0$ & - & 40 & 0 & 13 \\
$C D R=0.5$ & - & 40 & 0 & 9 \\
$C D R=1$ & - & 30 & 2 & 11 \\
$C D R=2$ & - & 44 & 4 & 14 \\
$C D R=3$ & - & 146 & 1 & 56 \\
\hline
\end{tabular}

Mayo Mayo Clinic, MSBB Mount Sinai Brain Bank, AD Alzheimer's disease, $P A$ pathological aging, $C D R$ Clinical Dementia Rating for available samples

Technologies). The RIN is determined by the software on the Bioanalyzer taking into account the entire electrophoretic trace of the RNA including the presence or absence of degradation products. The DV200 value is defined as the percentage of nucleotides $>200 \mathrm{nt}$. RIN and DV200 for all the samples can be found on Additional file 1: Table S1. The yield of each sample is determined by the Quant-iT RNA Assay (Life Technologies) on the Qubit Fluorometer (Fisher Scientific). The complementary DNA (cDNA) library was prepared with the TruSeq Stranded Total RNA Sample Prep with Ribo-Zero Gold kit (Illumina) and then sequenced by HiSeq 4000 (Illumina) using $2 \times 150$ paired-end reads at McDonnell Genome Institute, Washington University in St. Louis with a mean of $58.14 \pm 8.62$ million reads. Number of reads and other quality control (QC) metrics can be found in Additional file 1: Table S1.

\section{Mayo Clinic Brain Bank}

Mayo Clinic Brain Bank RNA-seq was accessed from the Advanced Medicines Partnership - Alzheimer's Disease (AMP-AD) portal (synapse ID $=5,550,404$; accessed January 2017) (Table 1). Paired end reads of $2 \times 101$ base pairs were generated by Illumina HiSeq 2000 sequencers for an average of 134.9 million reads per sample. Neuropathology criteria, quality control procedures, RNA extraction, and sequencing details are explained elsewhere [18].

RNA-seq based transcriptome data were generated from post-mortem brain tissue collected from cerebellum (CB; 189 samples) and temporal cortex (TC; 191 samples) of Caucasian subjects [18, 38]. RNA was extracted using Trizol $^{\circ}$ reagent and cleaned with Qiagen
RNeasy. RIN measurement was performed with Agilent Technologies 2100 Bioanalyzer. Samples with RIN > 5 were included. Library was prepared by Mayo Clinic Medical Genome Facility Gene Expression and Sequencing Cores with TruSeq RNA Sample Prep Kit (Illumina).

\section{Mount Sinai Brain Bank}

The Mount Sinai Brain Bank (MSBB) RNA-seq study was downloaded from the AMP-AD portal (synapse ID $=3,157,743$; accessed January 2017) (Table 1). Single-end reads of $100 \mathrm{nt}$ were generated by Illumina HiSeq 2500 System (Illumina, San Diego, CA, USA) for an average of 38.7 million reads per sample [39].

This dataset contains 1030 samples collected from four post-mortem brain regions of 300 subjects: anterior prefrontal cortex (APC; BA10); superior temporal gyrus (STG; BA22); parahippocampal gyrus (PHG; BA36); and inferior frontal gyrus (IFG; BA44). RNA-seq was generated using the TruSeq RNA Sample Preparation Kit v2 and Ribo-Zero rRNA removal kit (Illumina, San Diego, CA, USA) [39].

\section{Induced pluripotent stem cell (iPSC)-derived neurons}

Dermal fibroblasts were obtained from skin biopsies from research participants in the Knight-ADRC (Fibroblast lines: F11362, F12455, and F13504). Human fibroblasts were reprogrammed into iPSCs using non-integrating Sendai virus carrying OCT3/4, SOX2, KLF4, and cMYC $[40,41]$. iPSCs were manually selected and expanded on Matrigel in mTesR1 (StemCell Techologies). iPSCs were characterized for expression of pluripotency markers by immunocytochemistry and quantitative polymerase chain reaction (qPCR). qPCR with probes specific to the Sendai virus were used to confirm the absence of virus in the isolated clones. All cell lines were confirmed to have a normal karyotype based on G-band karyotyping. To generate cortical neurons, iPSCs were plated in a v-bottom plate in neural induction media (StemCell Technologies; 65,000 per well) to form highly uniform neural aggregates. After five days, neural aggregates were transferred onto PLO/laminin-coated tissue culture plates. Neural rosettes formed over 5-7 days. The resulting neural rosettes were then isolated by enzymatic selection (StemCell Technologies) and cultured as neural progenitor cells (NPCs). NPCs were then differentiated by culturing in neural maturation medium (neurobasal medium supplemented with B27, GDNF, BDNF, cAMP). RNA was collected from the cells and sequenced following the same protocol and processing pipeline as the DIAN and Knight-ADRC dataset.

In addition, we accessed RNA-seq data generated for iPSC-derived neurons from the Broad iPSC study [42] (synapse ID: syn3607401). Forebrain neurons from wild-type background were generated using an embryoid body-based protocol to produce neural progenitor cells 
(day 17) and mature neurons (days 57 and 100). RNA was purified using a PureLink RNA mini-kit (Life Technologies). RNA-seq libraries were prepared using Illumina Strand Specific TruSeq protocol and sequenced to obtain an average of $75 \mathrm{M}$ reads in paired reads per sample.

\section{Translating ribosome affinity purification (TRAP)-seq mice}

All animal procedures were performed in accordance with the guidelines of Washington University's Institutional Animal Care and Use Committee. The Rosa26 $6^{\text {fsTRAP }}$ mice (Gt(ROSA)26Sor ${ }^{\text {tm1(CAG-EGFP/Rpl10a,-birA)Wtp) }}$ [43] (The Jackson Laboratory) were crossed with $\mathrm{PV}^{\text {Cre }}$ mice

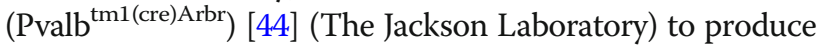
PV-TRAP mice directing expression of EGFP-L10a ribosomal fusion protein in parvalbumin (PV) expressing cells.

Purification of cell-type-specific messenger RNA (mRNA) by TRAP was described previously [45] with modifications. Briefly, PV-TRAP mouse brain was removed and quickly washed in ice-cold dissection buffer (1× HBSS, $2.5 \mathrm{mM}$ HEPES-KOH (pH 7.3), $35 \mathrm{mM}$ glucose, and $4 \mathrm{mM}$ $\mathrm{NaHCO}_{3}$ in $\mathrm{RNase}$-free water). Barrel cortex was rapidly dissected and flash-frozen in liquid nitrogen, and then stored at $-80{ }^{\circ} \mathrm{C}$ until use. Affinity matrix was prepared with $150 \mu \mathrm{L}$ of Streptavidin MyOne T1 Dynabeads, $60 \mu \mathrm{g}$ of Biotinylated Protein L, and $25 \mu \mathrm{g}$ of each of GFP antibodies 19C8 and 19F7. The tissue was homogenized on ice in $1 \mathrm{~mL}$ of tissue-lysis buffer (20 mM HEPES $\mathrm{KOH}$ (pH 7.4), $150 \mathrm{mM} \mathrm{KCl,} 10 \mathrm{mM} \mathrm{MgCl}$, EDTA-free protease inhibitors, $0.5 \mathrm{mM}$ DTT, $100 \mu \mathrm{g} / \mathrm{mL}$ cycloheximide, and $10 \mu \mathrm{L} / \mathrm{mL}$ rRNasin and Superasin). Homogenates were centrifuged for $10 \mathrm{~min}$ at $2000 \times g, 4{ }^{\circ} \mathrm{C}$, and $1 / 9$ sample volume of $10 \% \mathrm{NP}-40$ and $300 \mathrm{mM}$ DHPC were added to the supernatant at final concentration of $1 \%$ (vol/vol). After incubation on ice for $5 \mathrm{~min}$, the lysate was centrifuged for $10 \mathrm{~min}$ at $20,000 \times g$ to pellet insolubilized material. Then $200 \mu \mathrm{L}$ of freshly resuspended affinity matrix was added to the supernatant and incubated at $4{ }^{\circ} \mathrm{C}$ for $16-18 \mathrm{~h}$ with gentle end-over-end mixing in a tube rotator. After incubation, the beads were collected with a magnet and resuspended in $1000 \mu \mathrm{L}$ of high-salt buffer (20 mM HEPES $\mathrm{KOH}$ (pH 7.3), $350 \mathrm{mM} \mathrm{KCl,} 10 \mathrm{mM} \mathrm{MgCl} 2,1 \% \mathrm{NP}-40$, $0.5 \mathrm{mM}$ DTT, and $100 \mu \mathrm{g} / \mathrm{mL}$ cycloheximide) and collected with magnets as above. After four times of washing with high-salt buffer, RNA was extracted using Absolutely RNA Nanoprep Kit (Agilent Technologies) following the manufacturer's instructions. RNA quantification was measured using Qubit RNA HS Assay Kit (Life Technologies) and the integrity was determined by Bioanalyzer 2100 using an RNA Pico chip (Agilent Technologies). The cDNA library was prepared with Clontech SMARTer and then sequenced by HiSeq3000. Single-end reads of 50 base pairs were generated for an average of 29.2 million reads per sample (24 samples).

\section{iPSC-derived microglia}

The data were accessed from the AMP-AD portal (synapse ID: syn7203233). This dataset comprises iPSC-derived microglia $(n=10)$ from human primitive streak-like cells [46]. Within 30 days of differentiation, myeloid progenitors co-expressing CD14 and CX3CR1 were generated. These iPSC-derived microglia were able to perform phagocytosis and elicit ADP-induced intracellular $\mathrm{Ca}^{2+}$ transients that asserted their microglia identity as opposed to macrophage. Single-ended RNA-seq data were generated with the Illumina HiSeq 2500 platform following the Illumina protocol.

\section{RNA-seq QC and alignment}

FastQC was applied to DIAN and Knight-ADRC RNA-seq data to perform quality checks on various aspects of sequencing quality [47]. The DIAN and Knight-ADRC dataset was aligned to human GRCh37 primary assembly using Star (ver 2.5.2b) [48]. We used the primary assembly and aligned reads to the assembled chromosomes, un-localized and unplaced scaffolds, and discarded alternative haploid sequences. Sequencing metrics, including coverage, distribution of reads in the genome [49], ribosomal and mitochondrial contents, and alignment quality, were further obtained by applying Picard CollectRnaSeqMetrics (ver 2.8.2) to detect sample deviation. Additional QC metrics can be found in Additional file 1: Table S1.

Aligned and sorted bam files were loaded into IGV [50] to perform visual inspection of target variants. Samples carrying unexpected variants or missing expected variants were labeled as potential swapped samples. In addition, variants were called from RNA-seq following BWA/GATK pipeline $[51,52]$. The identity of the samples was later verified by performing IBD analysis against genomic typing from genome-wide association study chipsets.

\section{Expression quantification}

We applied Salmon transcript expression quantification (ver 0.7.2) [53] to infer the gene expression for all samples included in the reference panel and participants in the Mayo, MSBB, DIAN, and Knight-ADRC. We quantified the coding transcripts of Homo sapiens included in the GENCODE reference genome (GRCh37.75). Similarly, we quantified the expression of the mice samples included in the reference panel using the Mus musculus reference genome $(\mathrm{mm} 10)$.

\section{Reference panel}

\section{Reference samples}

We assembled a cell-type-specific reference panel from publicly available RNA-seq datasets comprising both immunopanning collected or iPSC-derived neurons, astrocytes, oligodendrocytes, and microglial cells from human and murine samples. For immunopanning collected 
cells, antibodies for cell-type-specific antigens were utilized to bind and immobilize their targeted cell types in order to immunoprecipitate and purify each cell type from the suspensions [30]. cDNA synthesis was accomplished using Ovation RNA-seq system V2 (Nugen 7102) and library prepared with Next Ultra RNA-seq library prep kit from Illumina (NEB E7530) and NEBNext $^{\circ}$ multiplex oligos from Illumina (NEB E7335 E7500). TruSeq RNA Sample Prep Kit (Illumina) was used to prepare library for paired-end sequence on $100 \mathrm{ng}$ of total RNA extracted from each sample. Illumina HiSeq 2000 Sequencer was used to sequence all libraries [30].

Both human adult TC tissue, collected from patients receiving neurological surgeries, and mice cells were disassociated, sorted and sequenced as described elsewhere [31], and deposited in the Gene Expression Omnibus GSE73721 and GSE52564. We also accessed neural progenitor cells (day 17) and mature human neurons (days 57 and 100) from Broad iPSC deposited in the AMP-AD portal [42] and neural progenitor cells and iPSC-derived neurons from [54]. Broad iPSC-derived neurons accessed from the AMP-AD portal were generated using an embryoid body-based protocol to differentiate into forebrain neurons. Wild-type cells used in the protocol were obtained from UConn StemCell Core. RNA was purified using PureLink RNA mini-kit (Life Technologies) and libraries were prepared by Broad Institute's Genomics Platform using TruSeq protocol. Please refer to Additional file 1: Table S2 for additional information.

\section{Marker genes}

The reference panel was assembled with samples from four distinct cell types. A redundant set of well-known cell-type markers was selected from the literature [31, 55, 56] (Additional file 1: Table S3). Principal component analysis (PCA) was performed on the reference panel using $\mathrm{R}$ function prcomp (version 3.3.3) to verify that the expressions of these gene were clustering samples by their cell types (Additional file 1: Figure S1b; Additional file 1: Figure S2a).

\section{Inference of the cellular population structure}

We ascertained alternative computation deconvolution algorithms implemented in the CellMix package (ver 1.6). Based on accuracy and robustness evaluation results, we compared and reported the following three algorithms that outperformed the others: Digital Sorting Algorithm (named "DSA") [27], which employs linear modeling to infer cell distributions; the method population-specific expression analysis (PSEA, also named meanProfile in CellMix implementation) [29] that calculates estimated expression profiles relative to the average of the marker gene list for each cell type [29]; and a semi-supervised learning method that employs non-negative matrix factorization (ssNMF in CellMix implementation) [57]. We employed a leave-one-out cross-validation (LOOCV) procedure to evaluate the accuracy provided by each method. The best performing algorithm ssNMF integrates cell-type marker genes to resolve the drawbacks of completely unsupervised standard non-negative matrix factorization. We followed the standard procedure described in the CellMix package, which included the extraction of marker genes from the reference samples (function extractMarkers from the CellMix package), and the posterior invocation of the function ged to infer cellular population from the gene expression of bulk RNA-seq data. Besides, we tested additional methods which provided considerably lower accuracy (least-squares fit [58], quadratic programing [59]) or no significant difference (support vector regression [26] or latent variable analysis [60]) to the methods presented.

We selected the reference samples that provide the most faithful transcriptomic profile for their respective cell types by following a LOOCV approach. We trained iteratively deconvolution models using all but one of the samples that was tested. Only samples predicted with a composition $>80 \%$ were kept for the reference panel (Additional file 1: Table S2; Additional file 1: Figure S2b).

\section{Accuracy and robustness evaluation Chimeric validation}

To emulate heterogeneous tissue with known and controlled cellular composition, we generated chimeric libraries pooling reads (to a total of 400,000) contributed from the human reference samples (see Additional file 1: Table S2). This process was repeated 720 times, using alternative reference samples to model each cell type. The proportion of reads that the libraries of neurons, astrocytes, oligodendrocytes, and microglia provided to the chimeric libraries varied in predefined ranges (Additional file 1: Figure S3). As a result, each of the chimeric libraries contained reads that followed 32 different distributions (neuronal reads contributed $2-36 \%$ of reads, astrocytes $22-76 \%$, oligodendrocytes 6-62\%, and microglia 1-5\%). Refer to Additional file 1: Table S4 for detailed description of the 32 different distributions. We quantified the chimeric reads using Salmon (v0.7.2) [53] and employed the reference samples that did not contribute reads to the chimeric library as reference panel for the deconvolution methods.

Overall, we quantified the expression of 23,040 (720 $\times$ 32) chimeric libraries. We evaluated the accuracy using the root-mean-square error (RMSE, Eq. 1 to compare the digital deconvolution cellular proportion estimates 
(method ssNMF) vs the defined proportion of reads specific to each of the chimeric libraries:

$$
R M S E=\sqrt{\frac{\sum_{i=1}^{n}(\hat{y} i-y i)^{2}}{n}}
$$

\section{$\hat{y} i$-estimated value, yi-observed value}

We also tested whether the deconvolution results were dominated by the expression of any specific marker gene and ascertained the robustness of the inferred cellular population structure to any possibly altered expression of marker genes. To do so, we performed the deconvolution analysis discarding each of the marker genes one at a time and evaluated how these distributions differed in comparison to the full gene reference panel.

\section{Statistical analysis}

We employed linear regression models to test the association between cell-type proportions and disease status ( $\mathrm{R}$ Foundation for Statistical Computing, ver.3.3.3). We used stepwise discriminant analysis (stepAIC function of $R$ package MASS, version 7.3-45) to determine significant covariates and to correct for confounding effects. We included RIN, batch, age at death, and post-mortem interval (PMI) as covariates for the Mayo Clinic analyses. For MSBB analyses, we corrected for RIN, PMI, race, batch, and age at death. We also used linear-mixed models to perform multiple-region association analysis, employing random slopes and random intercepts grouping by observations and by donors [61], and correcting for the same covariates previously described.

To analyze the DIAN and Knight-ADRC studies, we applied linear-mixed models (function lmer and Anova, $\mathrm{R}$ packages lme4 ver.1.1 and car ver.2.1, respectively), clustering at family level to ascertain the effect of the neuropathological status in the cell proportion and corrected for RIN and PMI. For late-onset specific analyses we also corrected for age at death.

Cellular composition shown as proportions were plotted using R package ggplot2 (ver 2.2.1).

\section{Results}

\section{Study design}

To infer cellular composition from RNA-seq, we first assembled a reference panel to model the transcriptomic signature of neurons, astrocytes, oligodendrocytes, and microglia. The panel was created by analyzing expression data from purified cell lines. We evaluated alternative digital deconvolution methods and selected the best performing for our primary analyses. We tested the digital deconvolution accuracy on iPSC-derived neurons/microglia cells and neuronal TRAP-seq (Fig. 1).
Finally, we verified its accuracy by creating artificial admixture with pre-defined cellular proportions.

Once the deconvolution approach was optimized, we calculated the cell proportion in $\mathrm{AD}$ cases and controls from the different brain regions of Mayo and MSBB datasets. The RNA-seq data for the Mayo Clinic study $(n=191)$ [18] and MSBB $(n=300)$ [39] are deposited in the AMP-AD knowledge portal (synapse ID: syn5550404 and syn3157743; Table 1). The Mayo study includes RNA-seq from the $\mathrm{TC}$ and $\mathrm{CB}$ for $\mathrm{AD}$ affected and non-demented controls, in addition to pathological aging (PA) participants (Fig. 1). The MSBB also profiled four additional cerebral cortex areas: APC; STG; PGH; and IFG; Table 1 and Fig. 1). We restricted the case-control analysis to subjects with definite $\mathrm{AD}$ and autopsy-confirmed controls. In addition, we generated RNA-seq from parietal lobe for participants of the Knight-ADRC (84 late-onset cases, carriers of genetic risk factors and 16 controls; Additional file 1: Table S1) and The Dominantly Inherited Alzheimer Network (DIAN; 19 carriers of mutations in APP, PSEN1, PSEN2) (Table 1; Fig. 1). We employed the same pipeline to process all of the samples in order to avoid any bias. Furthermore, RNA-seq from the Knight-ADRC and DIAN studies allowed us to compare the cell composition from ADAD vs LOAD brains, and similarly to test for differences in brains of controls, sporadic AD who do not carry any known high-risk variant, carriers of high-risk variants in TREM2 $(n=20)$, PLD3 $(n=33)$, and APOE \&4 allele.

\section{Development of a reference panel to estimate brain cellular population structure}

Due to limited availability of brain cell-type-specific transcriptomic data, we compiled reference samples from different sources, including single-population RNA-seq from mice and human (immunopan-purified oligodendrocytes, neurons, astrocytes and microglia, and iPSC-derived neurons and astrocytes).

We first tried to create a transcriptome-wide reference panel by selecting the genes that are differentially expressed among cell types [26, 60, 62]. However, the species heterogeneity of the reference samples we compiled ruled out this attempt, as the PCA showed that differences between the human and mice donor samples dominated the transcriptome-wide profiles (Additional file 1: Figure S1a). For this reason, we curated a list of marker genes that have been described to tag these distinct cell types $[31,55,56]$ (Additional file 1: Table S3). A visual inspection of the expression of these marker genes in the samples we compiled suggested a divergent transcriptomic profile among the cell types (Additional file 1: Figure S2a). The PCA showed that their expression was sufficient to cluster samples of neurons, astrocytes, oligodendrocytes, and microglia with their respective cell types, regardless of 


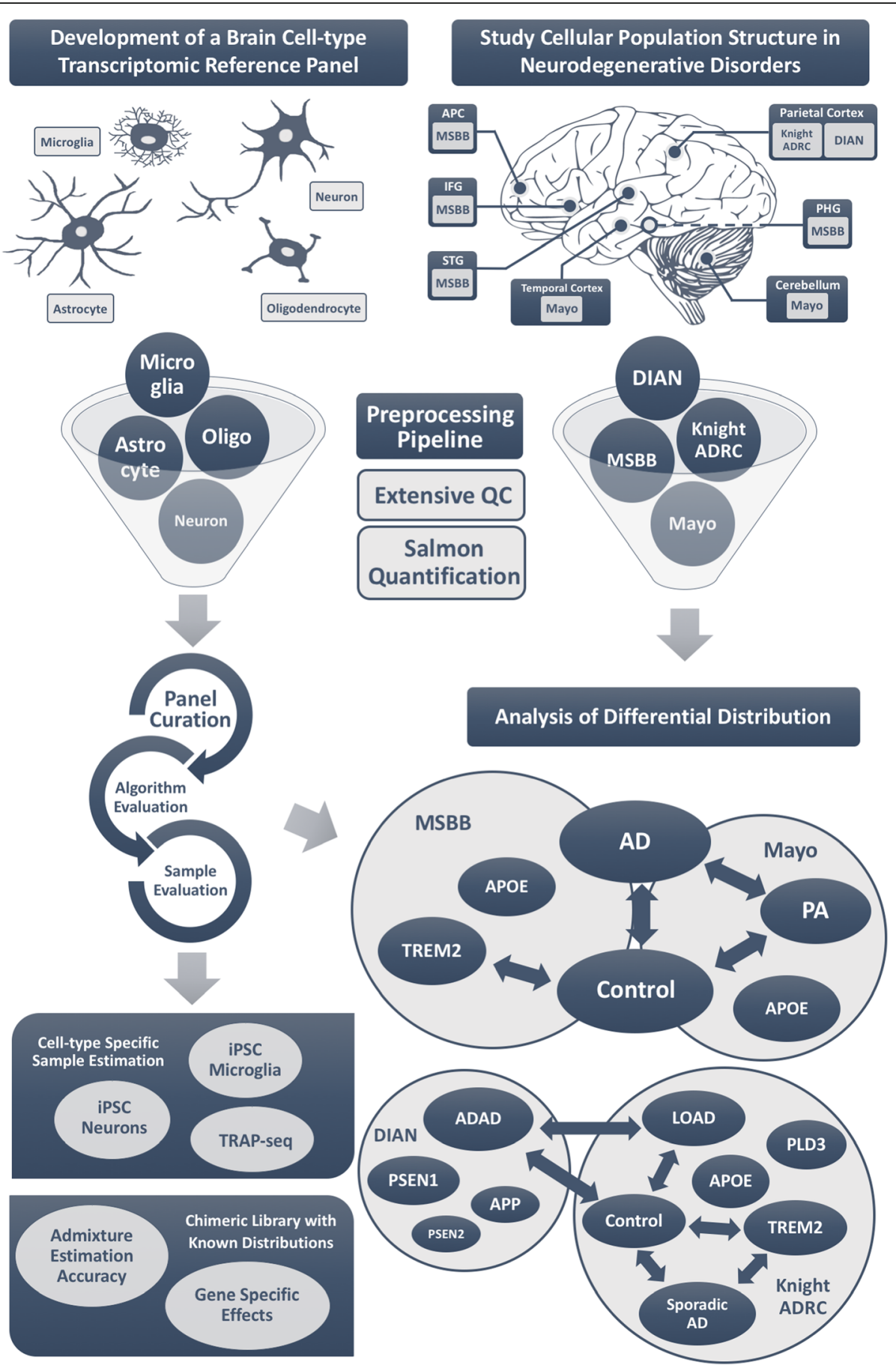

Fig. 1 (See legend on next page.) 


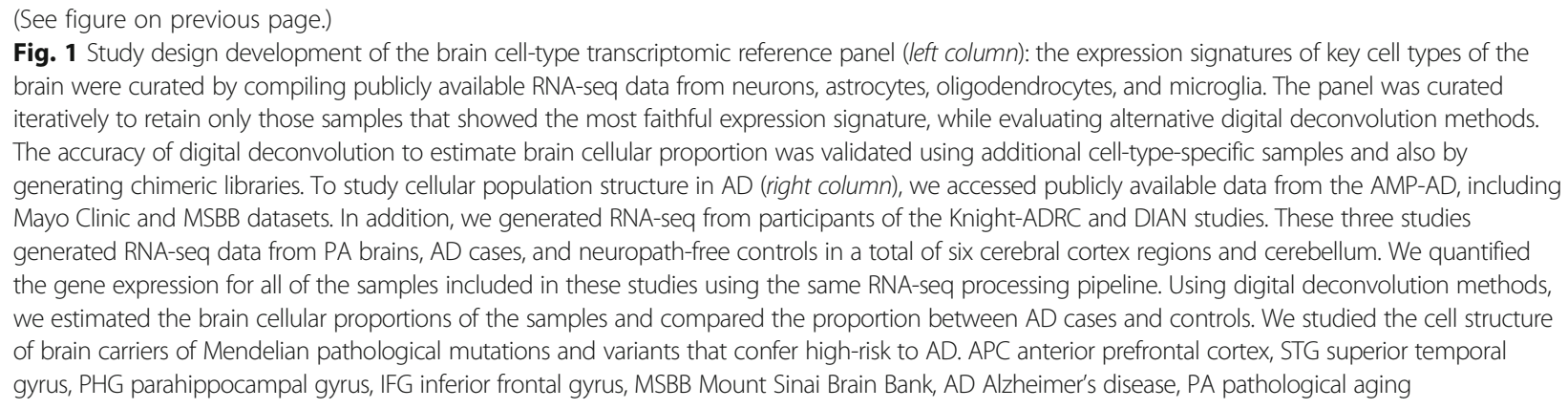

the species of the reference samples (Additional file 1: Figure S1b; Additional file 1: Table S2). We observed that some samples did not cluster with their expected cell types and coincidently the LOOCV indicated that these samples had an expression signatures that differed from the other samples of the same cell type. However, we found that all of these outliers correspond to samples not correctly purified or that were sequenced in early stages of differentiation (Additional file 1: Supplementary Results). After discarding these samples, we assessed six digital deconvolution algorithms implemented in the CellMix package [62] and found that the ssNMF [57] calculated the most accurate estimates (see "Methods"). Our final reference panel (Additional file 1: Table S2; Additional file 1: Table S3) had a very high confidence to predict cell types with a mean predicted accuracy $=95.2 \%$, s.d. $=4.3$ (Additional file 1: Figure S2b), and a RMSE $=0.06$ (Additional file 1: Table S5).

\section{Optimization, validation, and accuracy estimation of the reference panel and digital deconvolution method}

Once we identified the optimal approach to perform digital deconvolution from brain RNA-seq, we benchmarked it by using three sets of independent pure cell populations and simulated chimeric libraries.

We first validated the accuracy to predict neuronal composition by generating RNA-seq for eight iPSC-derived cortical neurons (see "Methods"). We observed an accurate prediction in these independent cell lines (mean neuronal proportion $=94.8 \%$, s.d. $=1.1 \%$; Additional file 1 : Figure S4a). We also ascertained the cellular composition of mRNA extracted from the barrel cortex neurons isolated by TRAP in 24 mice. TRAP is a method that captures cell-type-specific mRNA translation by purifying tagged ribosomal subunit and capturing the mRNA it bound to [45]. We observed an average of neuronal proportion $=96.7 \%$ and s.d. $=1.2 \%$ (Additional file 1: Figure S4b). Similarly, we assessed the RNA-seq data generated for iPSC-derived microglia $(n=10)$ deposited in the AMP-AD portal (synapse ID: syn7203233) and inferred their cellular population structure and observed a mean microglia proportion $=86.6 \%$ and s.d. $=7.1 \%$ (Additional file 1: Figure S4c).

To evaluate the accuracy of digital deconvolution for measuring cell-type proportion from cell-type admixtures, we simulated RNA-seq libraries by pooling reads from individual cell types into well-defined proportions. We combined randomly sampled reads from neurons, astrocytes, oligodendrocytes, and microglia to create chimeric libraries that mimic bulk RNA-seq from brain, but with a range of pre-defined cell-type distributions (Additional file 1: Figure S3). We then quantified the gene expression for the chimeric libraries and inferred the cell-type distribution (employing for the reference panel samples that did not contribute reads to the chimeric libraries). This process was repeated 23,040 times, choosing distinct human samples to represent each cell type and varying the proportions in 32 alternative distributions (see "Methods" and Additional file 1: Table S4). The overall error (RMSE) compared to known proportions $=0.08$.

Finally, we evaluated whether any gene included in the reference panel was dominating the inference of cell proportions. We re-calculated the cell-type distributions of the chimeric libraries but dropping each of the genes from the reference panel one at a time. We observed a negligible difference between the cellular population structure inferred using the full reference and the gene-dropped panels (average RMSE $=0.022$, s.d. $<0.01$ ) . In this way, we verified that the proportions inferred using the reference panel are not driven by the expression of a single gene. This reassured us the inference should be robust to any bias introduced by the potential association of a single gene included in the reference panel with a particular trait.

\section{Deconvolution of bulk RNA-seq of non-demented and AD brains shows a characteristic signature for neurodegeneration}

Pathologically, AD is associated with neuronal death and gliosis specifically in the cerebral cortex. We evaluated whether we could exploit deconvolution methods using our reference panel to detect altered cellular population 
structure from the bulk RNA-seq and whether this corresponded to known pathological alterations.

We initially analyzed the RNA-seq from the Mayo Clinic Brain Bank that includes bulk RNA-seq from the TC and CB for 191 participants [18] (Table 1). In the TC, we observed a significant higher astrocyte relative proportion $\left(\beta=0.23 ; p=5.01 \times 10^{-09}\right.$; Table 2; Fig. 2; Additional file 1: Table S6) in AD brains compared to control brains. We also found a significant lower relative proportion of neurons ( $\beta=-0.17 ; p=1.58 \times 10^{-07}$; Table 2; Fig. 2; Additional file 1: Table S6) and oligodendrocytes $(\beta=-0.07 ; p=1.8 \times$ $10^{-02}$; Table 2; Additional file 1: Figure S5; Additional file 1: Table S6). As expected given the absence of pathology, we did not observe a significant difference in the cell-type composition in the CB (Table 2).

The distribution of microglia was similar in the TC and $\mathrm{CB}$ from $\mathrm{AD}$ and control brains (Table 2; Additional file 1: Figure S5). The proportion of microglia was lower than any other cell types. The Mayo dataset also includes brains from individuals with PA (Table 1); which is neuropathologically defined by amyloid-beta $(\mathrm{A} \beta)$ senile plaque deposits but little or no neurofibrillary tau pathology $[18,63]$. We observed a significant lower relative proportion of microglia in PA brains compared to $\mathrm{AD}$ in both $\mathrm{TC}$ and $\mathrm{CB}$ (Additional file 1: Table S7; Additional file 1: Figure S6). Therefore, we speculated that the lack of changes in the $\mathrm{AD}$ microglial population was neither due to low statistical power nor the inability of our method to estimate the microglial proportions but reflected unaltered neuropathological observations in $\mathrm{AD}$ brains.

We also analyzed data from the MSBB, which contains bulk RNA-seq for four additional cerebral cortex areas (APC, STG, PHG, IFG). Replicating our findings from the Mayo dataset, we observed a significant lower relative proportion in neurons and increase in astrocytes in all four areas (Table 2; Fig. 2; and Additional file 1: Table S6). The strongest effect size was detected in the PHG and STG $\left(p<3.49 \times 10^{-07}\right)$ (Table 2; Additional file 1: Table S8). Neuropathological studies have described that the PHG is one of the first brain areas in which AD pathology occurs [64-66]. We also observed a significant and strong correlation between neuronal and astrocyte relative proportions and the last ascertained clinical status (CDR), the number of amyloid plaques and Braak staging (Table 2; Fig. 2; Additional file 1: Figure S7).

\section{The cellular population structure differs between ADAD vs LOAD}

While the loss of neurons is a common feature of AD, it is not clear whether the mechanism holds true across different forms of AD or AD cases carrying different genetic risk variants. Therefore, we investigated whether AD with distinct etiologies showed different cellular compositions. We generated RNA-seq data from the parietal lobe of participants enrolled in Knight-ADRC (84 LOAD, 3 ADAD, and 16 neuropath-free controls) and DIAN (19 ADAD) studies (Table 1; Additional file 1: Table S1). We selected the LOAD and ADAD participants to match for CDR at death, brain weight, and sex distributions (see Additional file 1: Table S1).

Using digital deconvolution, we determined the cellular composition for these brains. We observed a significant lower relative proportion of neurons $(\beta=-$ $0.02, p=2.66 \times 10^{-02}$ ) and significant higher relative proportion of astrocyte in $\mathrm{AD}(\beta=0.03, p=5.48 \times$ $10^{-03}$ ) for the combined LOAD and ADAD brains compared to controls (Table 3; Fig. 3; Additional file 1: Table S9), consistent with our findings in the Mayo and MSBB datasets. Similarly, the joint analysis of the brains from Knight-ADRC and DIAN showed significant associations between the neuronal and astrocyte relative proportions and neuropathological measures (Braak staging: $\beta=-0.03, p=8.51 \times 10^{-06}$ for neurons and $\beta=0.03, p=3.83 \times 10^{-06}$ for astrocytes; Table 3; Fig. $3 \mathrm{~b})$ as well as for clinical measures (CDR: $\beta=-$ $0.02, p=2.66 \times 10^{-02}$ for neurons and $\beta=0.03$ and $p=$ $5.48 \times 10^{-03}$ for astrocytes; Table 3; Fig. 3c). We did not observe a significant difference in the compositions of microglia or oligodendrocytes (Table 3; Additional file 1: Figure S8).

Next, we compared the cell proportion of LOAD vs ADAD and found that the cell composition differs between them. We first selected the LOAD brains $(n=25)$ to match the Braak staging distribution of ADAD brains $(n=17)$. The ADAD brains showed a significant lower relative neuronal proportion compared to LOAD brains $\left(\beta=-0.08 ; p=1.03 \times 10^{-02}\right.$; Table 3$)$ and an increased relative astrocyte proportion $\left(\beta=0.11 ; p=9.26 \times 10^{-04}\right.$; Table 3). Then, we analyzed the entire Knight-ADRC LOAD brains, by extending the model to correct for Braak stages. We also observed significant lower relative neuronal proportion $\left(\beta=-0.09 ; p=4.71 \times 10^{-03}\right.$; Table 3; Fig. 3a; Additional file 1: Table S9) and increased relative astrocyte proportion $\left(\beta=0.11 ; p=5.24 \times 10^{-04}\right.$; Table 3; Fig. 3a; Additional file 1: Table S9) in ADAD brains compared to LOAD. We observed the same cellular differences when we corrected for CDR at death $(\beta=-$ $0.12 ; p=2.11 \times 10^{-03}$ for neurons and $\beta=0.13 ; p=$ $6.29 \times 10^{-04}$ for astrocytes; Table 3; Fig. 3b, c). In summary, our results indicate that ADAD individuals present a higher neuronal loss even in the same stage of the disease, suggesting that in ADAD neuronal death plays a more important role in pathogenesis compared to sporadic $\mathrm{AD}$, in which other factors such as inflammation or immune response may be involved. 


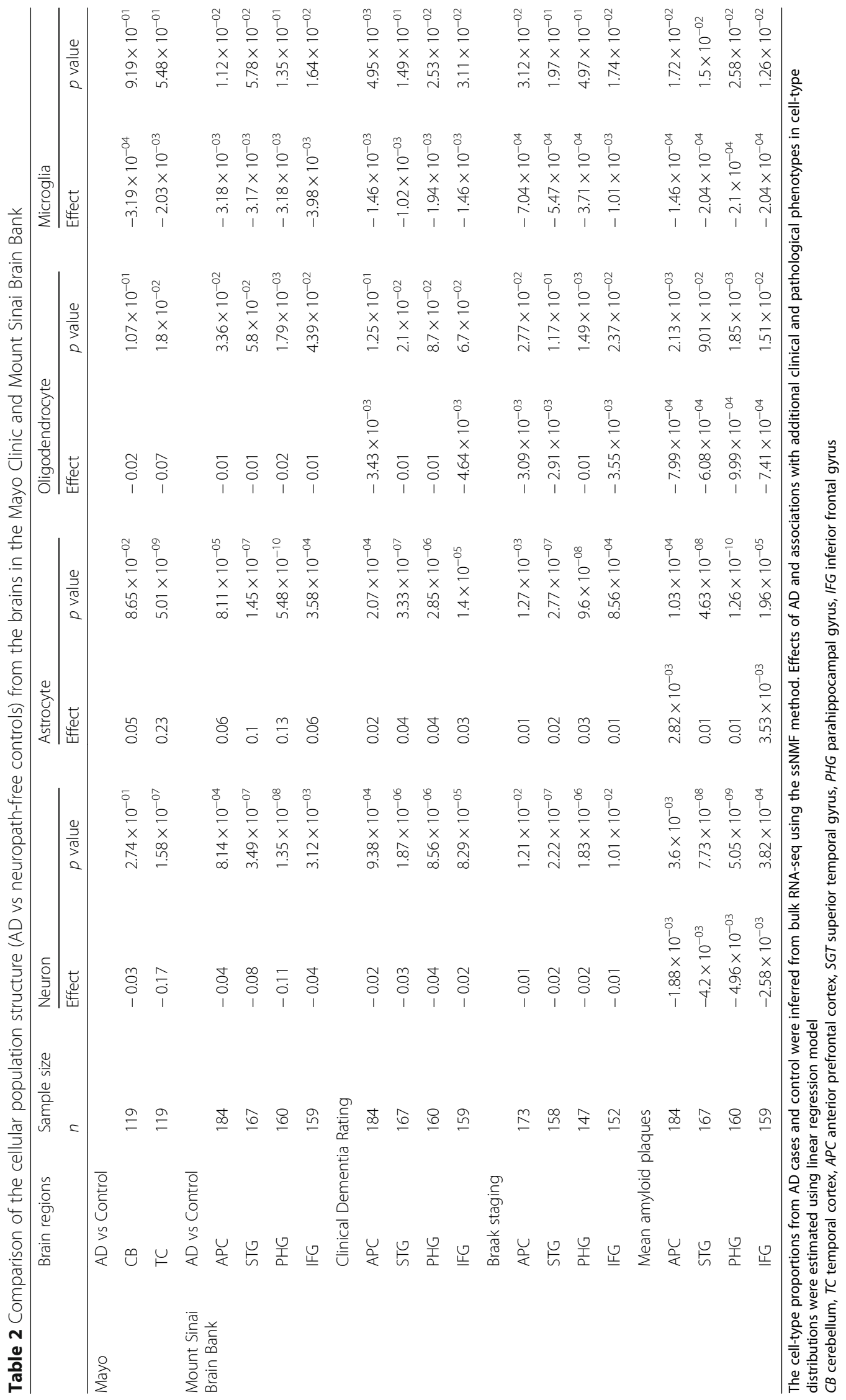




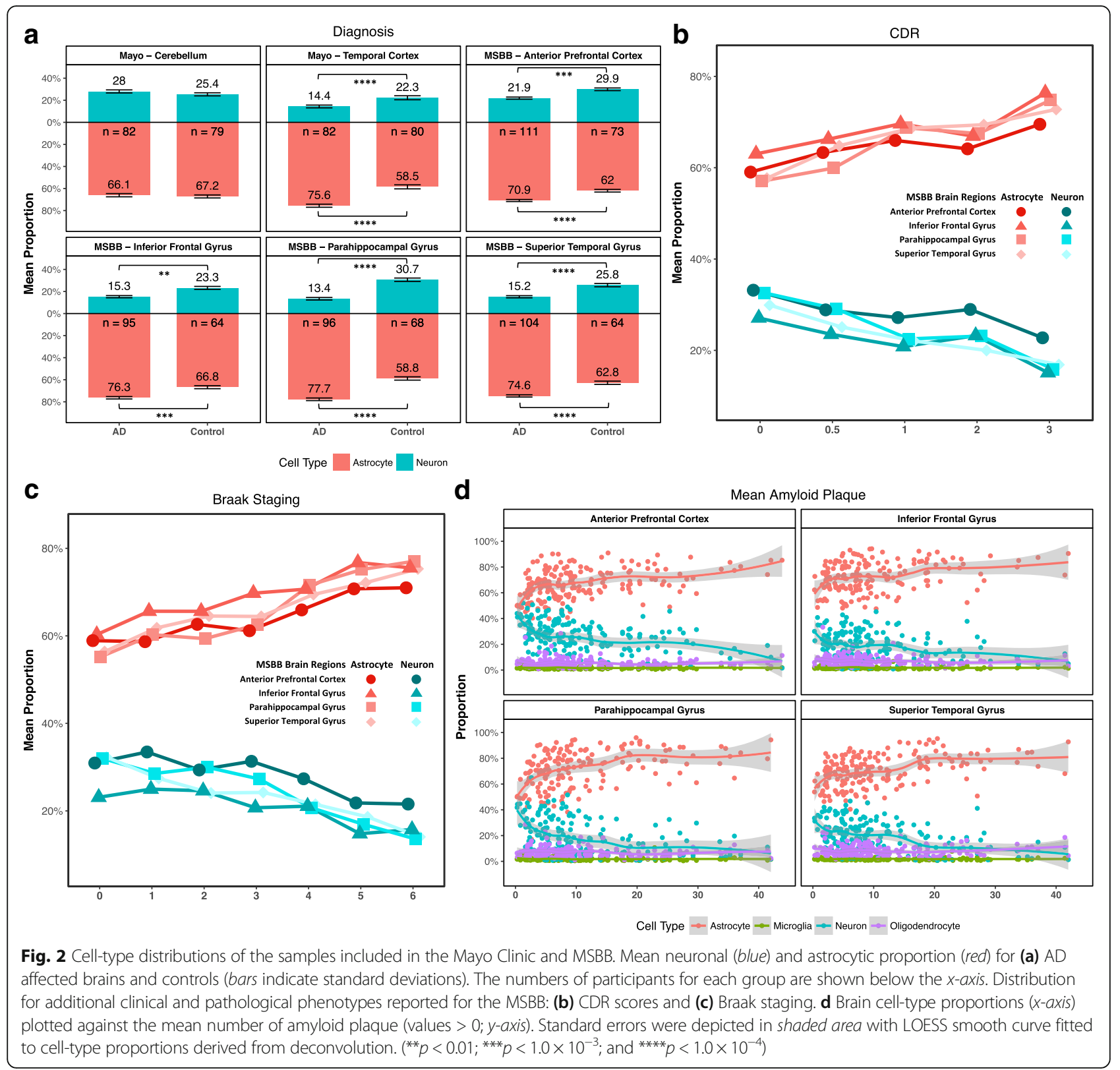

\section{Specific genetic variants confer a distinctive cell composition profile}

A variety of genetic variants increase risk of LOAD; however, it is unclear if the cellular mechanisms are the same across these distinct risk factors. Therefore, we tested the hypothesis that distinct genetic causes of LOAD have characteristic cellular population signatures.

We initially ascertained the effect of $A P O E \& 4$ on the cell-type composition. We observed a significant lower relative proportion of neurons $(\beta=-0.06$ for each of the $\varepsilon 4$ alleles; $p=9.91 \times 10^{-03}$ ) and increase of relative proportion of astrocytes $\left(\beta=0.10 ; p=4.15 \times 10^{-02}\right)$ from the TC included in the Mayo Clinic dataset (Additional file 1: Table S10; Fig. 4a; Additional file 1: Figure S9a). This finding was replicated when we performed a multi-area analysis of the MSBB dataset $\left(\beta=-0.04 ; p=2.60 \times 10^{-03}\right.$ and $\beta=0.05 ; p=1.31 \times 10^{-03}$ for neurons and astrocytes, respectively; Table 4; Fig. 4a; Additional file 1: Table S10; Additional file 1: Figure S9a). Given the strong risk conferred by the $A P O E \varepsilon 4$ allele [4], we studied its effects on the cell-type composition by restricting our analysis to AD brains. We observed a significant association in the multi-area analysis of the MSBB dataset $(\beta=-0.03 p=$ $4.01 \times 10^{-02}$; Table 4; Fig. 4b; Additional file 1: Table S11; Additional file 1: Figure S9b) and also a significant increase in relative proportion of astrocytes $(\beta=0.03$; $p=1.23 \times 10^{-02}$; Table 4; Fig. 4b; Additional file 1 : Table S11; Additional file 1: Figure S9b). We also 
Table 3 Cellular population structure altered in the parietal lobe from AD brains in the DIAN study and Knight-ADRC brain bank

\begin{tabular}{|c|c|c|c|c|c|c|c|c|c|}
\hline \multirow[t]{2}{*}{ Disease status } & \multirow{2}{*}{$\begin{array}{l}\text { Sample } \\
\text { size } \\
n\end{array}$} & \multicolumn{2}{|c|}{ Neuron } & \multicolumn{2}{|c|}{ Astrocyte } & \multicolumn{2}{|c|}{ Oligodendrocyte } & \multicolumn{2}{|l|}{ Microglia } \\
\hline & & Effect & $p$ value & Effect & $p$ value & Effect & $p$ value & Effect & $p$ value \\
\hline \multicolumn{10}{|l|}{ AD status } \\
\hline$A D^{a}$ vs Control & 122 & -0.11 & $5.52 \times 10^{-04}$ & 0.14 & $2.48 \times 10^{-05}$ & -0.03 & $6.5 \times 10^{-02}$ & $-2.64 \times 10^{-03}$ & $2.49 \times 10^{-01}$ \\
\hline ADAD vs Control & 38 & -0.19 & $3.94 \times 10^{-07}$ & 0.24 & $1.57 \times 10^{-10}$ & -0.04 & $8.5 \times 10^{-03}$ & -0.01 & $7.77 \times 10^{-05}$ \\
\hline LOAD vs Control & 100 & -0.09 & $5.67 \times 10^{-03}$ & 0.12 & $3.34 \times 10^{-04}$ & -0.02 & $1.06 \times 10^{-01}$ & $-1.70 \times 10^{-03}$ & $4.57 \times 10^{-01}$ \\
\hline \multicolumn{10}{|l|}{ ADAD vs LOAD } \\
\hline Braak matched & 42 & -0.08 & $1.03 \times 10^{-02}$ & 0.11 & $9.26 \times 10^{-04}$ & -0.03 & $7.1 \times 10^{-02}$ & $-1.46 \times 10^{-03}$ & $7.01 \times 10^{-01}$ \\
\hline Braak corrected & 91 & -0.09 & $4.71 \times 10^{-03}$ & 0.11 & $5.24 \times 10^{-04}$ & -0.02 & $1.77 \times 10^{-01}$ & $-2.41 \times 10^{-03}$ & $4.25 \times 10^{-01}$ \\
\hline CDR corrected & 94 & -0.12 & $2.11 \times 10^{-03}$ & 0.13 & $6.29 \times 10^{-04}$ & -0.02 & $3.8 \times 10^{-01}$ & $-3.11 \times 10^{-03}$ & $2.41 \times 10^{-01}$ \\
\hline \multicolumn{10}{|l|}{ Clinical Dementia Rating } \\
\hline$A D^{a}$ and Controls & 110 & -0.02 & $2.66 \times 10^{-02}$ & 0.03 & $5.48 \times 10^{-03}$ & -0.01 & $2 \times 10^{-01}$ & $-4.63 \times 10^{-04}$ & $4.77 \times 10^{-01}$ \\
\hline ADAD and Controls & 26 & -0.08 & $4.12 \times 10^{-04}$ & 0.11 & $1.78 \times 10^{-07}$ & 0.01 & $4.03 \times 10^{-03}$ & $-1.55 \times 10^{-03}$ & $1.75 \times 10^{-08}$ \\
\hline LOAD and Controls & 100 & -0.02 & $3.22 \times 10^{-02}$ & 0.03 & $7.01 \times 10^{-03}$ & -0.01 & $1.81 \times 10^{-01}$ & $-4.64 \times 10^{-04}$ & $5.11 \times 10^{-01}$ \\
\hline \multicolumn{10}{|l|}{ Braak staging } \\
\hline$A D^{a}$ and Controls & 106 & -0.03 & $8.51 \times 10^{-06}$ & 0.03 & $3.83 \times 10^{-06}$ & $-4.24 \times 10^{-03}$ & $2.04 \times 10^{-01}$ & $-2.52 \times 10^{-04}$ & $6.81 \times 10^{-01}$ \\
\hline ADAD and Controls & 33 & -0.05 & $2.37 \times 10^{-05}$ & 0.06 & $2.45 \times 10^{-05}$ & -0.01 & $2.29 \times 10^{-01}$ & $-7.2 \times 10^{-04}$ & $4.89 \times 10^{-01}$ \\
\hline LOAD and Controls & 88 & -0.03 & $7.41 \times 10^{-04}$ & 0.03 & $4.63 \times 10^{-04}$ & $-3.72 \times 10^{-03}$ & $3.29 \times 10^{-01}$ & $-1.66 \times 10^{-04}$ & $7.86 \times 10^{-01}$ \\
\hline
\end{tabular}

${ }^{\mathrm{a}} \mathrm{AD}$ includes both autosomal dominant $\mathrm{AD}$ (ADAD) and late-onset $\mathrm{AD}$ (LOAD)

The cellular population structure was inferred using the ssNMF method. Effects and $p$-values for the association with disease status, clinical dementia rating and

Braak staging using generalized mixed models. We identified similar trends with approximately the same significance levels

$A D$ Alzheimer's disease, $A D A D$ autosomal dominant $A D, L O A D$ late-onset $A D$
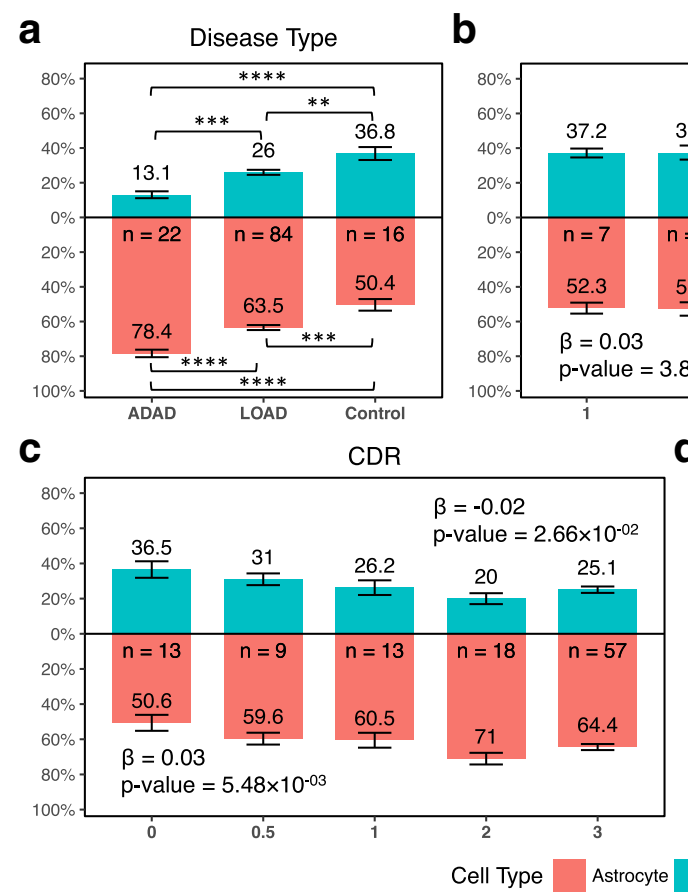

b

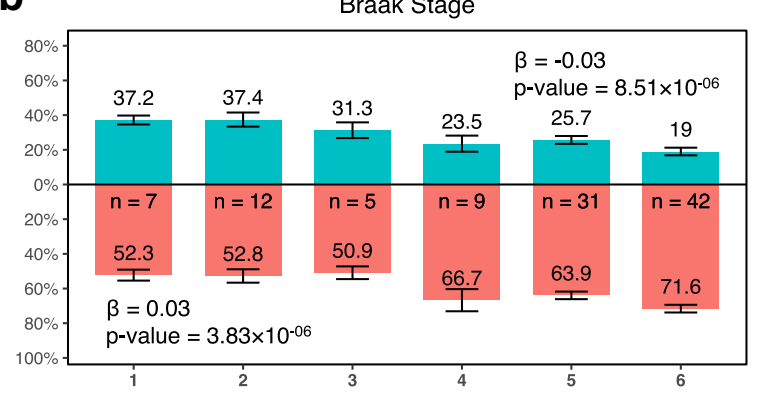

d

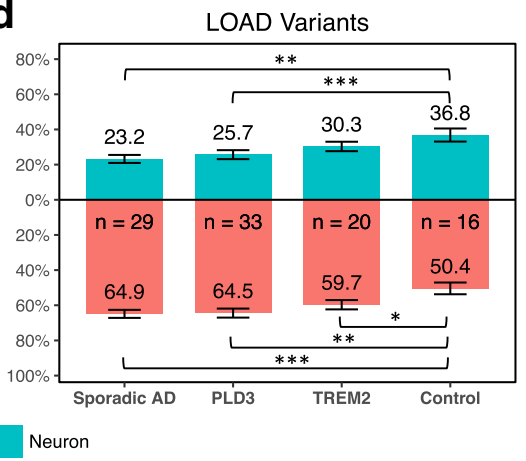

Fig. 3 Neuron and astrocyte distributions from the DIAN and Knight-ADRC brains. a Mean neuronal (blue) and astrocytic (red) proportions for carriers of pathogenic mutations in APP, PSEN1, or PSEN2 (ADAD), late-onset AD (LOAD), and neuropath-free controls (bars indicate standard deviations). Neuronal and astrocytic proportions plotted against (b) Braak staging and (c) by CDR. $\mathbf{d}$ Cell-type distributions for carriers of AD genetic risk factors. Lines indicate significance levels $\left({ }^{*} p<0.05 ;{ }^{* *} p<0.01 ;{ }^{* *} p<1.0 \times 10^{-3} ;{ }^{* * * *} p<1.0 \times 10^{-4}\right.$ ) 

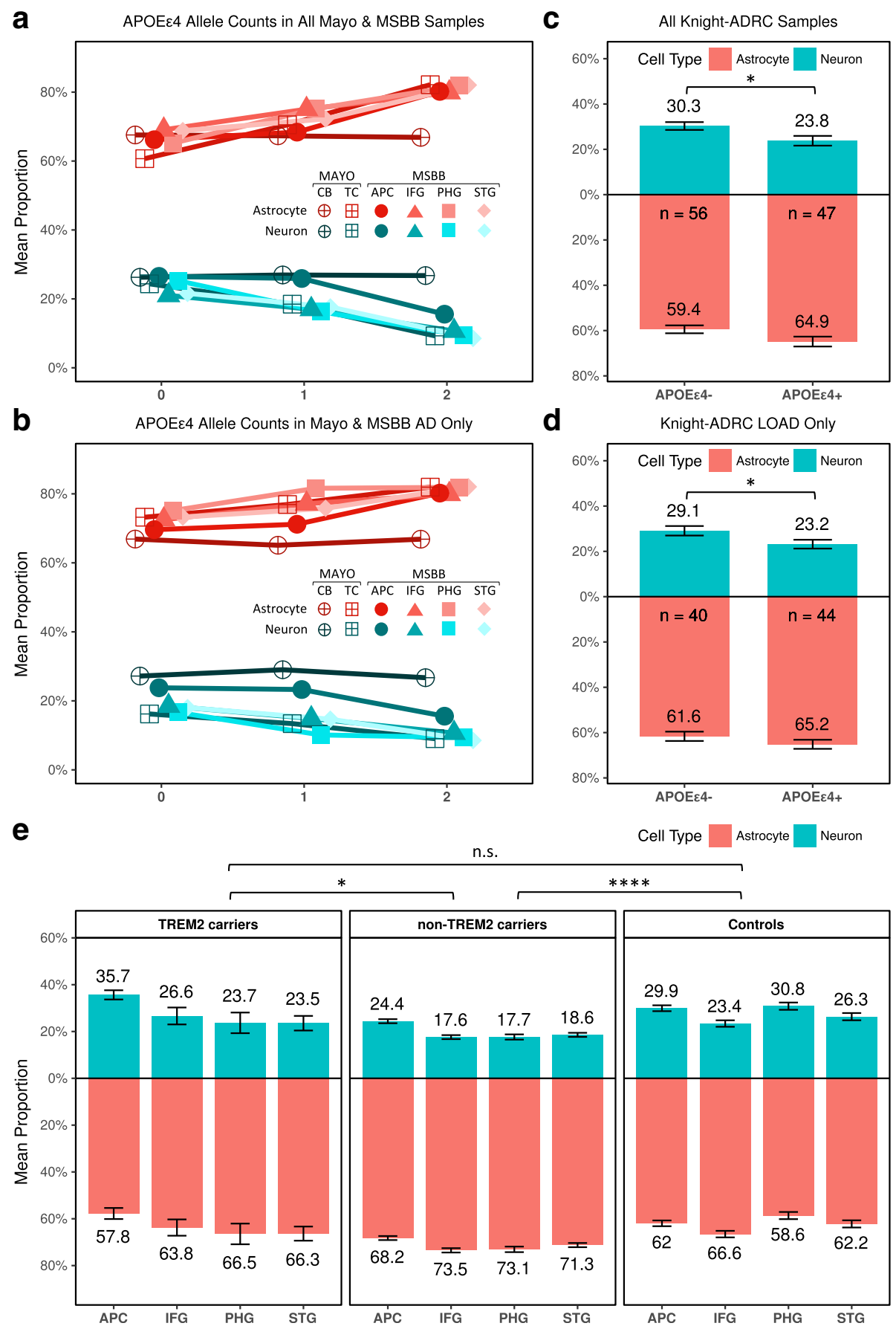

Fig. 4 Effect of the APOE $\varepsilon 4$ allele and TREM2 coding variants on the cellular population structure. Mean neuronal (blue) and astrocytic (red) proportions for (a) AD cases and controls in the Knight-ADRC brains categorized by APOE $\varepsilon 4$ carriers vs non-carriers and (b) AD cases of KnightADRC brain bank (bars indicate standard deviations). c AD cases and controls in the Mayo Clinic and MSBB. $\mathbf{d}$ AD cases in the Mayo Clinic and MSBB. e Neuronal (blue) and astrocyte (red) distributions for samples included in the MSBB stratified by TREM2 genetic status. APC anterior prefrontal cortex, STG superior temporal gyrus, PHG parahippocampal gyrus, IFG inferior frontal gyrus (n.s. $p>0.05 ;{ }^{*} p<0.05 ;{ }^{* * *} p<1.0 \times 10^{-4}$ )

observed a significant decrease in relative proportion of neurons $\left(\beta=-0.06 ; p=2.11 \times 10^{-02}\right.$; Table 4 ; Fig. $\left.4 \mathrm{c}\right)$ when we analyzed the LOAD and control brains from the Knight-ADRC. When we restricted the analysis to
AD brains from the Knight-ADRC and compared the $A P O E \& 4$ carriers $(n=44)$ to non-carriers $(n=40)$ we also observed a decreased relative neuronal proportion $\left(\beta=-0.06 ; p=2.69 \times 10^{-02}\right.$; Table $4 ;$ Fig. $\left.4 d\right)$. We 


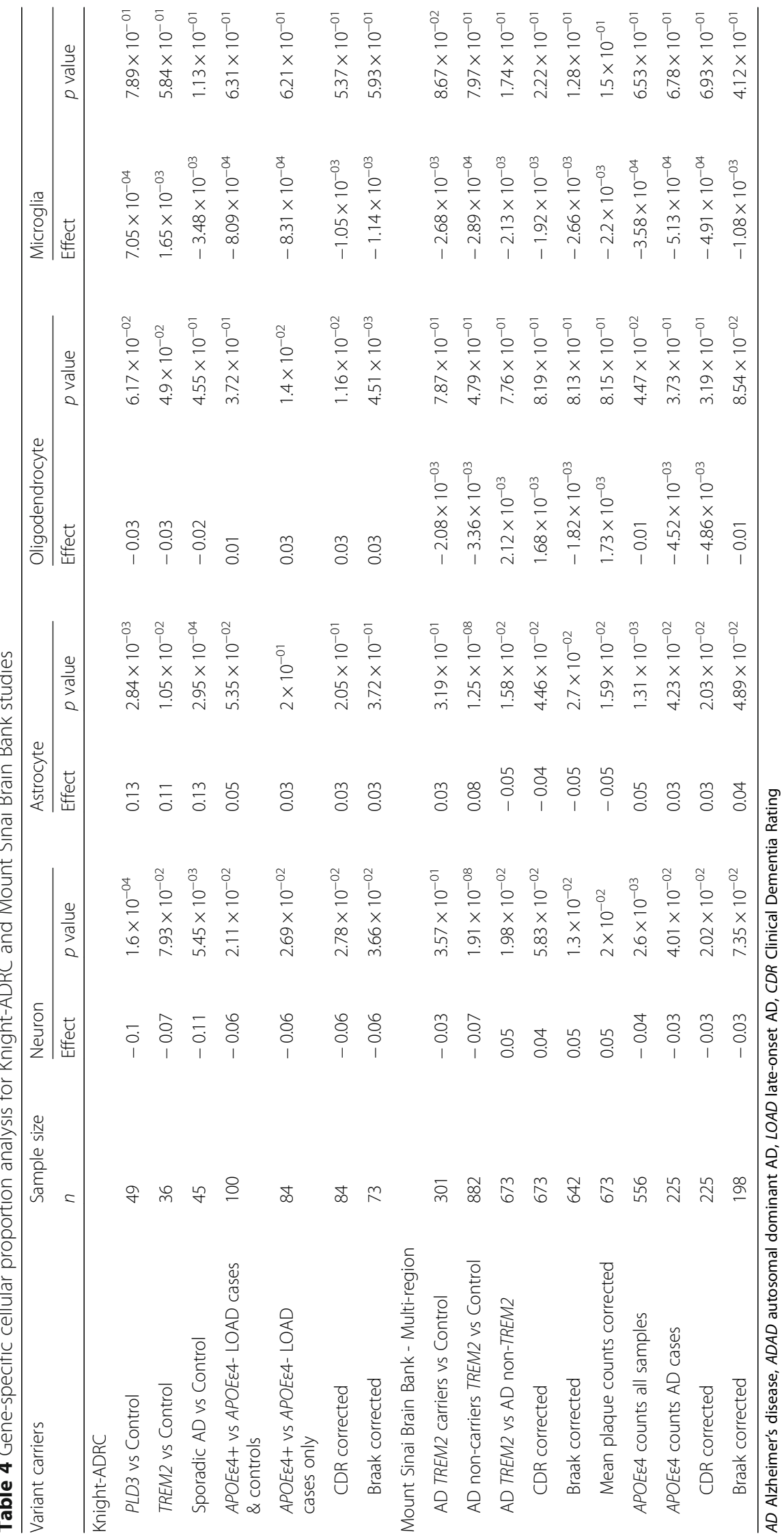


extended the models to correct for the Braak stages and observed a significant association for the relative proportion of neurons with the APOE $\varepsilon 4$ allele in the Knight-ADRC dataset $\left(\beta=-0.06 ; p=3.66 \times 10^{-02}\right.$; Table 4) and a significant association for the relative proportion of astrocytes in the MSBB $\left(\beta=0.04 ; p=4.89 \times 10^{-02}\right.$; Table 4$)$. Furthermore, we performed a meta-analysis to combine the evidence of both studies and observed a significant association of the relative neuronal proportion with $A P O E$ \&4 allele $\left(p=1.86 \times 10^{-02}\right)$ and marginally significant association for the relative astrocytic relative proportion $(p=0.09)$.

Next, we analyzed the cellular composition in PLD3 carriers $(n=33)$. PLD3 carriers exhibited significantly lower relative proportion of neurons compared to controls $\left(\beta=-0.10 ; p=1.60 \times 10^{-04}\right.$; Fig. $\left.3 \mathrm{~d}\right)$ and a significant higher relative proportion of astrocytes $(\beta=0.13$; $p=2.84 \times 10^{-03}$; Table 4; Fig. 3d). Sporadic AD non-carrier cases also exhibited significantly lower relative proportion of neurons compared to controls $(\beta=-$ $0.11 ; p=5.45 \times 10^{-03}$ ) and significant higher relative proportion of astrocytes $\left(\beta=0.13 ; p=2.95 \times 10^{-04}\right.$; Table 4; Fig. 3d). The cell proportion between sporadic AD non-carriers and PLD3 carriers did not show any significant difference $(p>0.05)$.

Finally, we performed similar analyses with TREM2 carriers. TREM2 is involved in the immune response and its role in amyloid- $\beta$ deposition or clearance remains controversial [67]. Our analysis on the Knight-ADRC data showed significantly higher relative astrocytic proportion in AD affected TREM2 carriers $(\mathrm{n}=20)$ compared to controls $\left(\beta=0.11 ; p=1.05 \times 10^{-02}\right.$; Table 4; Fig. 3d). Despite TREM2 carriers presenting lower neuron relative proportion compared to controls, this difference was not statistically significant ( $p>0.05$; Table 4 ; Fig. $3 \mathrm{~d}$ ). We analyzed whether the TREM2 carriers provided sufficient power to detect a significant association. Our empirical estimates showed that TREM2 sample size provides $96 \%$ of power to detect an association with an effect size comparable to that observed for sporadic $\mathrm{AD}(\beta=-0.11)$. We also investigated the cellular proportion of the 11 TREM2 carriers in the MSBB dataset. The multi-region analysis showed TREM2 carriers do not show a significant difference in relative neuronal proportion compared to controls ( $p>0.05$; Table 4; Fig. 4e), whereas in the AD TREM2 non-carriers the relative neuronal and astrocytic proportions are significantly different from controls $(\beta=-$ $0.07 ; \quad p=1.91 \times 10^{-08} ;$ and $\beta=0.08 ; \quad p=1.25 \times 10^{-08}$ respectively; Table 4; Fig. 4e).

In fact, our analyses indicate that TREM2 carriers have a unique cellular brain composition distinct than the other AD cases. TREM2 brains showed significantly higher relative neuronal proportion $\left(\beta=0.05 ; p=1.98 \times 10^{-02}\right)$ and significantly lower relative astrocyte proportion than the
$\mathrm{AD}$ non-carries $\left(\beta=-0.05 ; p=1.58 \times 10^{-02}\right.$; Table 4$)$. The distribution of $\mathrm{CDR}$, mean number of amyloid plaques, and Braak staging do not differ between strata. Nonetheless, we verified that the cellular proportions were still significantly different after correcting for each of those variables (Table 4). These results suggested that the mechanism that lead to disease in TREM2 carriers is less neuron-centric than in the general $\mathrm{AD}$ population.

\section{Discussion}

We have developed, optimized, and validated a digital deconvolution approach to infer cell composition from bulk brain gene expression that integrates publicly available cell-type specific expression data while addressing the heterogeneity of the phenotypic differences of samples and technical characteristics of transcriptome ascertainment. We acknowledge that the accuracy of this platform might be affected by the phenotypic diversity of the reference panel or the disease-induced dysregulation of genes it includes. However, the deconvolution approach proved to be robust to the genes included in the reference panel, as we demonstrated that the proportions it inferred are not driven by the expression of any single gene. This platform produced reliable cell proportion estimates, as was shown by the evaluation of independent datasets of iPSC-derived neurons and microglia, mice cortical neurons (Additional file 1: Figure S4), and simulated chimeric libraries.

We used this approach to deconvolve studies that include large numbers of neuropathologically defined $\mathrm{AD}$ and control brains with their transcriptome ascertained in distinct brain regions. We observed consistently significant lower relative neuronal proportion and increased relative astrocyte proportions in the cerebral cortex suggesting neuronal loss and astrocytosis. Compatible with other studies, we also identified that the altered cellular proportion is also significantly associated with decline in cognition and Braak staging [68]. In contrast, we did not identify a significant difference in the cellular population structure in the cerebellum, a region not affected in AD (Table 2; Fig. 2a).

We generated RNA-seq data from brains carrying pathogenic mutations in APP, PSEN1, and PSEN2, which cause alterations in $A \beta$ processing and lead to ADAD, and also generated RNA-seq from brains of LOAD and neuropath-free controls. We observed altered cell composition in both ADAD and LOAD compared to controls. However, we identified that ADAD brains have a different cell-type composition than disease-stage-matched LOAD, as the ADAD has a significantly lower relative neuronal proportion and more pronounced astrocytosis. Given the specific cellular population structure of the TREM2 carriers, we compared the neuronal and astrocytic relative proportion of ADAD to that of LOAD 
non-carriers of variants in TREM2 and observed significant differences $\left(\beta=-0.09\right.$ and $p=6.89 \times 10^{-03}$ for neurons and $\beta=0.10 ; p=1.49 \times 10^{-03}$ for astrocytes). This indicates that the difference of the relative proportion between ADAD and LOAD are not driven by TREM2 carrier brains. Based on our results, we would hypothesize that this change in $A \beta$ processing of ADAD would lead to more direct to neuronal death than the pathological processes of LOAD. Similarly, decreased neuronal and increased astrocyte relative proportions were significantly associated with $A P O E \& 4$ allele. It has been reported $A P O E \& 4$ allele increases the risk for AD by affecting APP metabolism or A $\beta$ clearance $[69,70]$, suggesting a direct link between APP metabolism and neuronal death.

In contrast, the analysis of the Knight-ADRC brains showed that the neuronal relative proportion decrease is less pronounced in TREM2 carriers than in other LOAD cases. We replicated this finding in a multi-area analysis from the MSBB dataset. These results may implicate that TREM2 risk variants lead to a cascade of pathological events that differ from those occurring in sporadic AD cases, which is also consistent with the known biology of TREM2. Further longitudinal neuroimaging analysis is required to validate our findings. TREM2 is involved in AD pathology through microglia mediated pathways, implicated on altered immune response and inflammation [71]. Recent studies in TREM2 knock-out animals showed that fewer microglia cells were found surrounding $A \beta$ plaques with impaired microgliosis [72]. Furthermore, TREM2 deficiency was reported to attenuate tauopathy against brain atrophy [73]. We found no significant difference in the proportion of microglia between AD cases and controls. However, we found significantly decreased microglia in brains exhibiting PA (Additional file 1: Table S7; Additional file 1: Figure S6), proving that these studies are sufficiently powered to identify significant differences. In any case, we cannot rule out the possibility of a change in the activation stage of microglia in these individuals. Overall, these results suggest that TREM2 affects AD risk through a slightly different mechanism to that of ADAD or LOAD in general. Therefore, other pathogenic mechanisms should contribute to disease. We believe that a detailed modeling of immune response cells, reflecting the alternative microglia activation states, will generate more accurate profiles to elucidate the immune cell distribution in $\mathrm{AD}$.

\section{Conclusions}

There is a large interest in the scientific community to use brain expression studies to try to identity novel pathogenic mechanisms in $\mathrm{AD}$ and to identify novel therapeutic targets. These efforts are generating a large amount of bulk RNA-seq data, as single-cell RNA
(scRNA-seq) from human brain tissue in large sample sizes is not feasible. Single-cell sorting needs to be performed with fresh tissue [74], which restrains the analysis of highly characterized fresh-frozen brains collected by AD research centers. Our results indicate that digital deconvolution methods can accurately infer relative cell distributions from brain bulk RNA-seq data, but we recognize the importance of obtaining traditional neuropathological measures to validate the results we observed. Having this approach validated for AD can have an important impact in the community, because digital deconvolution analyses can: (1) reveal distinct cellular composition patterns underlying different disease etiologies; (2) provide additional insights about the overall pathologic mechanisms underlying different mutations carriers for variants as in genes such as TREM2, APOE, APP, PSEN1, and PSEN2; (3) correct the effect that altered cell composition and genetic statuses have in addition to downstream transcriptomic analyses and lead to novel and informative results; and (4) help the analysis of highly informative frozen brains collected over the years.

In conclusion, our study provides a reliable approach to enhance our understanding of the fundamental cellular mechanisms involved in AD and enable the analysis of large bulk RNA-seq data that may lead to novel discoveries and insights into neurodegeneration.

\section{Additional file}

Additional file 1: Supplementary results, tables and figures. (DOCX $2353 \mathrm{~kb})$

\section{Abbreviations}

AD: Alzheimer's disease; ADAD: Autosomal dominant Alzheimer's disease; AMP-AD: Advanced Medicines Partnership - Alzheimer's disease;

APC: Anterior prefrontal cortex; A $\beta$ : Amyloid-beta; CB: Cerebellum; CDR: Clinical Dementia Rating; DIAN: Dominantly Inherited Alzheimer Network; DSA: Digital sorting algorithm; IFG: Inferior frontal gyrus; iPSC: Induced pluripotent stem cell; Knight-ADRC: Charles F. and Joanne Knight Alzheimer's Disease Research Center (Knight ADRC); LOAD: Late-onset Alzheimer's disease; meanProfile: Implementation of method PopulationSpecific Expression Analysis; MSBB: Mount Sinai Brain Bank; PA: Pathological aging; PCA: Principal component analyses; PHG: Parahippocampal gyrus; PSEA: Population-Specific Expression Analysis; RIN: RNA integrity number; RMSE: Root-mean-square error; ssNMF: Semi-supervised non-negative matrix factorization; STG: Superior temporal gyrus; TC: Temporal cortex; TRAPseq: Translating ribosome affinity purification sequencing

\section{Acknowledgements}

We thank all participants and their families for their commitment and dedication to helping advance research into the early detection and causation of AD; and the Knight-ADRC research and support staff at each of the participating sites for their contributions to this study.

This manuscript has been reviewed by DIAN Study investigators for scientific content and consistency of data interpretation with previous DIAN Study publications. We acknowledge the altruism of the participants and their families and contributions of the DIAN research and support staff at each of the participating sites for their contributions to this study.

We would like to thank the operations staff at the Elizabeth $\mathrm{H}$. and James S. McDonnell III Genome Institute at Washington University with their assistance 
in constructing the RNA-seq libraries and generating sequence data for our project. This work was also supported by accessing to equipment made possible by the Hope Center for Neurological Disorders and the Departments of Neurology and Psychiatry at Washington University School of Medicine. We also thank Allison M. Lake for her comments and suggestions.

The results published here are in whole or in part based on data obtained from the AMP-AD Knowledge Portal accessed at doi:https:// doi.org/10.7303/syn2580853. Mayo Clinic RNA-seq data were provided by the following sources: The Mayo Clinic Alzheimer's Disease Genetic Studies, led by Dr. Nilufer Taner and Dr. Steven G. Younkin, Mayo Clinic Jacksonville, FL using samples from the Mayo Clinic Study of Aging, the Mayo Clinic Alzheimer's Disease Research Center, and the Mayo Clinic Brain Bank. MSBB RNA-seq data were generated from postmortem brain tissue collected through the Mount Sinai VA Medical Center Brain Bank and were provided by Dr. Eric Schadt from Mount Sinai School of Medicine. MSSMiPSC data were generated by Kristen Brennand, a New York Stem Cell Foundation - Robertson Investigator.

\section{Funding}

This work was supported by grants from the National Institutes of Health (R01-AG044546, P01-AG003991, RF1AG053303, R01-AG035083, and R01NS085419) and the Alzheimer's Association (NIRG-11-200110). This research was conducted while CC was a recipient of a New Investigator Award in Alzheimer's disease from the American Federation for Aging Research. CC and CMK are recipient of a BrightFocus Foundation Alzheimer's Disease Research Grant (A2013359S). This work was supported in part by $\mathrm{NIH}$ K01AG046374 awarded to CMK, and also the Tau Consortium (CMK). JDD is supported by the Brain and Behavior Research Foundation and the $\mathrm{NIH}$ (R01NS102272). The recruitment and clinical characterization of research participants at Washington University were supported by NIH P50 AG005681, P01 AG003991, and P01 AG026276.

DIAN data collection and sharing for this project were supported by The Dominantly Inherited Alzheimer's Network (DIAN, U19AG032438) funded by the National Institute on Aging (NIA), the German Center for Neurodegenerative Diseases (DZNE), Raul Carrea Institute for Neurological Research (FLENI), Partial support by the Research and Development Grants for Dementia from Japan Agency for Medical Research and Development, AMED, and the Korea Health Technology R\&D Project through the Korea Health Industry Development Institute (KHIDI).

Mayo Clinic RNA-seq data collection was supported through funding by NIA grants P50 AG016574, R01 AG032990, U01 AG046139, R01 AG018023, U01 AG006576, U01 AG006786, R01 AG025711, R01 AG017216, R01 AG003949, NINDS grant R01 NS080820, CurePSP Foundation, and support from the Mayo Foundation. Study data include samples collected through the Sun Health Research Institute Brain and Body Donation Program of Sun City, Arizona. The Brain and Body Donation Program is supported by the National Institute of Neurological Disorders and Stroke (U24 NS072026 National Brain and Tissue Resource for Parkinson's Disease and Related Disorders), the National Institute on Aging (P30 AG19610 Arizona Alzheimer's Disease Core Center), the Arizona Department of Health Services (contract 211002, Arizona Alzheimer's Research Center), the Arizona Biomedical Research Commission (contracts 4001, 0011, 05-901, and 1001 to the Arizona Parkinson's Disease Consortium), and the Michael J. Fox Foundation for Parkinson's Research. MSSMiPSC data collection was supported by the Brain and Behavior Research Foundation, NIH grant R01 MH101454, and the New York Stem Cell Foundation. We analyzed iPSC-derived microglia RNA-seq data funded by NIH U01AG046170.

\section{Availability of data and materials}

The datasets analyzed during the current study are available from these sources: Mayo Clinic Brain Bank: https://www.synapse.org/ \#!Synapse:syn5550404; Mount Sinai Brain Bank: https:// www.synapse.org/\#!Synapse:syn3157743; GSE52564: https:// www.ncbi.nlm.nih.gov/geo/query/acc.cgi?acc=GSE52564; GSE73721: https://www.ncbi.nlm.nih.gov/geo/query/acc.cgi?acc=GSE73721; BroadiPSC: https://www.synapse.org/\#!Synapse:syn3607401; The KnightADRC: https://www.synapse.org/\#!Synapse:syn12181323.

According to the data request terms, DIAN data are available upon request: http://dian.wustl.edu.

\section{Authors' contributions}

ZL performed the analyses, contributed to the study design and data interpretation. JDA, UD, JB, RM, KB, QB, NC, JDD, JML, JCM, RJB, and CMK contributed by data collection, data processing, QC, and cleaning. CC and $\mathrm{OH}$ designed the study, collected data, supervised the analyses, performed data interpretation, and wrote the manuscript. All the authors read and provide input to the manuscript.

\section{Ethics approval and consent to participate}

All research participants contributing clinical, genetic, or tissue samples for genetic analysis to this study provided written informed consent, subject to oversight by the Washington University in St. Louis, Mayo clinic or Mount Sinai School of Medicine review boards. All procedures of the Knight-ADRC (201105102) and DIAN (201106339) studies were approved by the Washington University Human Research Protection Office and written informed consent was obtained from each participant. The study was conducted according to the principles of the Declaration of Helsinki. All animal procedures were performed in accordance with the guidelines of Washington University's Institutional Animal Care and Use Committee.

\section{Competing interests}

The authors declare that they have no competing interests.

\section{Publisher's Note}

Springer Nature remains neutral with regard to jurisdictional claims in published maps and institutional affiliations.

\section{Author details \\ ${ }^{1}$ Department of Psychiatry, Washington University School of Medicine, $660 \mathrm{~S}$. Euclid Ave. B8134, St. Louis, MO 63110, USA. ${ }^{2}$ Medical Scientist Training Program, Washington University School of Medicine, 660 S. Euclid Ave, St. Louis, MO 63110, USA. ${ }^{3}$ Department of Neurology, Washington University School of Medicine, 660 S. Euclid Ave, St. Louis, MO 63110, USA. ${ }^{4}$ Department of Pathology \& Immunology, Washington University in St. Louis, School of Medicine, 510 S. Kingshighway, MC 8131, Saint Louis, MO 63110, USA. ${ }^{5}$ Knight Alzheimer's Disease Research Center, Washington University School of Medicine, 660 S. Euclid Ave, St. Louis, MO 63110, USA. ${ }^{6}$ Hope Center for Neurological Disorders, Washington University School of Medicine, 660 S. Euclid Ave. B8111, St. Louis, MO 63110, USA. ${ }^{7}$ Department of Genetics, Washington University School of Medicine, 660 S. Euclid Ave, St. Louis, MO 63110, USA.}

\section{Received: 5 March 2018 Accepted: 15 May 2018}

\section{Published online: 08 June 2018}

\section{References}

1. LaFerla FM, Oddo S. Alzheimer's disease: Abeta, tau and synaptic dysfunction. Trends Mol Med. 2005;11:170-6.

2. De Strooper B, Annaert W. Novel research horizons for presenilins and gamma-secretases in cell biology and disease. Annu Rev Cell Dev Biol. 2010; 26:235-60.

3. Selkoe DJ. Alzheimer's disease: genes, proteins, and therapy. Physiol Rev. 2001;81:741-66.

4. Corder EH, Saunders AM, Strittmatter WJ, Schmechel DE, Gaskell PC, Small $\mathrm{GW}$, et al. Gene dose of apolipoprotein E type 4 allele and the risk of Alzheimer's disease in late onset families. Science. 1993;261:921-3.

5. Benitez BA, Cruchaga C. TREM2 and neurodegenerative disease. N Engl J Med. 2013:369:1567-8.

6. Guerreiro R, Wojtas A, Bras J, Carrasquillo M, Rogaeva E, Majounie E, et al. TREM2 variants in Alzheimer's disease. N Engl J Med. 2013;368:117-27.

7. Cruchaga C, Karch CM, Jin SC, Benitez BA, Cai Y, Guerreiro R, et al. Rare coding variants in the phospholipase D3 gene confer risk for Alzheimer's disease. Nature. 2014:505:550-4.

8. Steinberg $\mathrm{S}$, Stefansson $H$, Jonsson $T$, Johannsdottir $H$, Ingason A, Helgason $\mathrm{H}$, et al. Loss-of-function variants in ABCA7 confer risk of Alzheimer's disease. Nat Genet. 2015;47:445-7.

9. Del-Aguila JL, Fernandez MV, Jimenez J, Black K, Ma SM, Deming Y, Carrell D, Saef B, Howells B, Budde J, Cruchaga C. Role of ABCA7 loss-of-function variant in Alzheimer's disease: a replication study in European-Americans. Alzheimers Res Ther. 2015;7:73. 
10. Rogaeva E, Meng Y, Lee JH, Gu Y, Kawarai T, Zou F, et al. The neuronal sortilin-related receptor SORL1 is genetically associated with Alzheimer disease. Nat Genet. 2007;39:168-77.

11. Fernandez MV, Black K, Carrell D, Saef B, Budde J, Deming Y, et al. SORL1 variants across Alzheimer's disease European American cohorts. Eur J Hum Genet. 2016;24:1828-30.

12. Tang M, Ryman DC, MCDade E, Jasielec MS, Buckles VD, Cairns NJ, et al. Neurological manifestations of autosomal dominant familial Alzheimer's disease: a comparison of the published literature with the Dominantly Inherited Alzheimer Network observational study (DIAN-OBS). Lancet Neurol. 2016;15:1317-25.

13. Ryan NS, Nicholas JM, Weston PS, Liang Y, Lashley T, Guerreiro R, et al. Clinical phenotype and genetic associations in autosomal dominant familial Alzheimer's disease: a case series. Lancet Neurol. 2016;15:1326-35.

14. Padurariu M, Ciobica A, Mavroudis I, Fotiou D, Baloyannis S. Hippocampal neuronal loss in the CA1 and CA3 areas of Alzheimer's disease patients. Psychiatr Danub. 2012;24:152-8.

15. Wright AL, Zinn R, Hohensinn B, Konen LM, Beynon SB, Tan RP. Neuroinflammation and neuronal loss precede Abeta plaque deposition in the hAPP-J20 mouse model of Alzheimer's disease. PLoS One. 2013;8:e59586.

16. Holtzman DM, Morris JC, Goate AM. Alzheimer's disease: the challenge of the second century. Sci Transl Med. 2011;3:77sr71.

17. Golub VM, Brewer J, Wu X, Kuruba R, Short J, Manchi M. Neurostereology protocol for unbiased quantification of neuronal injury and neurodegeneration. Front Aging Neurosci. 2015;7:196.

18. Allen M, Carrasquillo MM, Funk C, Heavner BD, Zou F, Younkin CS, et al. Human whole genome genotype and transcriptome data for Alzheimer's and other neurodegenerative diseases. Sci Data. 2016;3:160089.

19. Narayanan M, Huynh JL, Wang K, Yang X, Yoo S, McElwee J, et al. Common dysregulation network in the human prefrontal cortex underlies two neurodegenerative diseases. Mol Syst Biol. 2014;10:743.

20. Srinivasan K, Friedman BA, Larson JL, Lauffer BE, Goldstein LD, Appling LL, et al. Untangling the brain's neuroinflammatory and neurodegenerative transcriptional responses. Nat Commun. 2016;7:11295.

21. Zhang B, Gaiteri C, Bodea LG, Wang Z, McElwee J, Podtelezhnikov AA, et al. Integrated systems approach identifies genetic nodes and networks in lateonset Alzheimer's disease. Cell. 2013;153:707-20.

22. Chan G, White CC, Winn PA, Cimpean M, Replogle JM, Glick LR, et al. CD33 modulates TREM2: convergence of Alzheimer loci. Nat Neurosci. 2015;18: 1556-8.

23. Miller JA, Woltjer RL, Goodenbour JM, Horvath S, Geschwind DH. Genes and pathways underlying regional and cell type changes in Alzheimer's disease. Genome Med. 2013;5:48

24. Parikshak NN, Gandal MJ, Geschwind DH. Systems biology and gene networks in neurodevelopmental and neurodegenerative disorders. Nat Rev Genet. 2015;16:441-58.

25. Gaiteri C, Mostafavi S, Honey CJ, De Jager PL, Bennett DA. Genetic variants in Alzheimer disease - molecular and brain network approaches. Nat Rev Neurol. 2016;12:413-27.

26. Newman AM, Liu CL, Green MR, Gentles AJ, Feng W, Xu Y. Robust enumeration of cell subsets from tissue expression profiles. Nat Methods. 2015;12:453-7

27. Zhong Y, Wan YW, Pang K, Chow LM, Liu Z. Digital sorting of complex tissues for cell type-specific gene expression profiles. BMC Bioinformatics. 2013;14:89.

28. Shen-Orr SS, Gaujoux R. Computational deconvolution: extracting cell typespecific information from heterogeneous samples. Curr Opin Immunol. 2013;25:571-8

29. Kuhn A, Thu D, Waldvogel HJ, Faull RL, Luthi-Carter R. Population-specific expression analysis (PSEA) reveals molecular changes in diseased brain. Nat Methods. 2011;8:945-7.

30. Zhang Y, Chen K, Sloan SA, Bennett ML, Scholze AR, O'Keeffe S, et al. An RNA-sequencing transcriptome and splicing database of glia, neurons, and vascular cells of the cerebral cortex. J Neurosci. 2014;34:11929-47.

31. Zhang Y, Sloan SA, Clarke LE, Caneda C, Plaza CA, Blumenthal PD, et al. Purification and characterization of progenitor and mature human astrocytes reveals transcriptional and functional differences with mouse. Neuron. 2016:89:37-53.

32. Brennand KJ, Simone A, Jou J, Gelboin-Burkhart C, Tran N, Sangar S, et al. Modelling schizophrenia using human induced pluripotent stem cells. Nature 2011, 473:221-225.
33. Knight-Alzheimer's Disease Research Center [http://alzheimer.wustl.edu/].

34. Dominantly Inherited Alzheimer Network [https://dian.wustl.edu//].

35. Mirra SS, Heyman A, McKeel D, Sumi SM, Crain BJ, Brownlee LM, et al. The Consortium to Establish a Registry for Alzheimer's Disease (CERAD). Part II. Standardization of the neuropathologic assessment of Alzheimer's disease. Neurology. 1991:41:479-86.

36. Morris JC. Clinical dementia rating: a reliable and valid diagnostic and staging measure for dementia of the Alzheimer type. Int Psychogeriatr. 1997;9(Suppl 1):173-6. discussion 177-178

37. Braak H, Braak E. Staging of Alzheimer's disease-related neurofibrillary changes. Neurobiol Aging. 1995;16:271-8. discussion 278-284

38. AMPAD Knowledge Portal Mayo Clinic RNAseq [https://www.synapse.org/ \#!Synapse:syn5550404].

39. AMPAD Knowledge Portal Mount Sinai Brain Bank RNAseq [https://www. synapse.org/\#!Synapse:syn3157743].

40. Takahashi K, Yamanaka S. Induction of pluripotent stem cells from mouse embryonic and adult fibroblast cultures by defined factors. Cell. 2006;126:663-76.

41. van de Leemput J, Boles NC, Kiehl TR, Corneo B, Lederman P, Menon V, et al. CORTECON: a temporal transcriptome analysis of in vitro human cerebral cortex development from human embryonic stem cells. Neuron. 2014;83:51-68.

42. UConn StemCell Core Broad iPSC deposited in the AMP-AD [https://www. synapse.org/\#!Synapse:syn3607401].

43. Zhou P, Zhang Y, Ma Q, Gu F, Day DS, He A, et al. Interrogating translational efficiency and lineage-specific transcriptomes using ribosome affinity purification. Proc Natl Acad Sci U S A. 2013;110:15395-400.

44. Hippenmeyer S, Vrieseling E, Sigrist M, Portmann T, Laengle C, Ladle DR, et al. A developmental switch in the response of DRG neurons to ETS transcription factor signaling. PLoS Biol. 2005;3:e159.

45. Heiman M, Kulicke R, Fenster RJ, Greengard P, Heintz N. Cell type-specific mRNA purification by translating ribosome affinity purification (TRAP). Nat Protoc. 2014:9:1282-91.

46. Douvaras P, Sun B, Wang M, Kruglikov I, Lallos G, Zimmer M, et al. Directed differentiation of human pluripotent stem cells to microglia. Stem Cell Reports. 2017;8:1516-24.

47. Andrews S. FastQC: a quality control tool for high throughput sequence data. 2010. Available online at http://www.bioinformatics.babraham.ac.uk/ projects/fastqc.

48. Dobin A, Davis CA, Schlesinger F, Drenkow J, Zaleski C, Jha S, et al. STAR: ultrafast universal RNA-seq aligner. Bioinformatics. 2013;29:15-21.

49. Broad Institute The Picard Pipeline [http://broadinstitute.github.io/picard/].

50. Robinson JT, Thorvaldsdottir H, Winckler W, Guttman M, Lander ES, Getz G, et al. Integrative genomics viewer. Nat Biotechnol. 2011;29:24-6.

51. Li H, Durbin R. Fast and accurate short read alignment with BurrowsWheeler transform. Bioinformatics. 2009;25:1754-60.

52. McKenna A, Hanna M, Banks E, Sivachenko A, Cibulskis K, Kernytsky A, et al. The Genome Analysis Toolkit: a MapReduce framework for analyzing nextgeneration DNA sequencing data. Genome Res. 2010;20:1297-303.

53. Patro R, Duggal G, Love MI, Irizarry RA, Kingsford C. Salmon provides fast and bias-aware quantification of transcript expression. Nat Methods. 2017; 14:417-9.

54. Brennand KJ. The hiPSC Neurons and NPCS study (MSSMiPSC) deposited in the AMP-AD. https://www.synapse.org/\#!Synapse:syn5986884

55. Cahoy JD, Emery B, Kaushal A, Foo LC, Zamanian JL, Christopherson KS, et al. A transcriptome database for astrocytes, neurons, and oligodendrocytes: a new resource for understanding brain development and function. J Neurosci. 2008;28:264-78.

56. Holtman IR, Raj DD, Miller JA, Schaafsma W, Yin Z, Brouwer N, et al. Induction of a common microglia gene expression signature by aging and neurodegenerative conditions: a co-expression meta-analysis. Acta Neuropathol Commun. 2015;3:31.

57. Gaujoux R, Seoighe C. Semi-supervised Nonnegative Matrix Factorization for gene expression deconvolution: a case study. Infect Genet Evol. 2012;12:913-21.

58. Abbas AR, Wolslegel K, Seshasayee D, Modrusan Z, Clark HF. Deconvolution of blood microarray data identifies cellular activation patterns in systemic lupus erythematosus. PLoS One. 2009;4:e6098.

59. Gong T, Hartmann N, Kohane IS, Brinkmann V, Staedtler F, Letzkus M, et al. Optimal deconvolution of transcriptional profiling data using quadratic programming with application to complex clinical blood samples. PLoS One. $2011 ; 6:$ e27156 
60. Chikina M, Zaslavsky E, Sealfon SC. CellCODE: a robust latent variable approach to differential expression analysis for heterogeneous cell populations. Bioinformatics. 2015;31:1584-91.

61. Sul JH, Han B, Ye C, Choi T, Eskin E. Effectively identifying eQTLs from multiple tissues by combining mixed model and meta-analytic approaches. PLoS Genet. 2013;9:e1003491.

62. Gaujoux R, Seoighe C. CellMix: a comprehensive toolbox for gene expression deconvolution. Bioinformatics. 2013;29:2211-2.

63. Murray ME, Dickson DW. Is pathological aging a successful resistance against amyloid-beta or preclinical Alzheimer's disease? Alzheimers Res Ther. 2014;6:24.

64. Echavarri C, Aalten P, Uylings HB, Jacobs HI, Visser PJ, Gronenschild EH, et al. Atrophy in the parahippocampal gyrus as an early biomarker of Alzheimer's disease. Brain Struct Funct. 2011;215:265-71.

65. Braak H, Braak E. Neurofibrillary changes confined to the entorhinal region and an abundance of cortical amyloid in cases of presenile and senile dementia. Acta Neuropathol. 1990;80:479-86.

66. Van Hoesen GW, Augustinack JC, Dierking J, Redman SJ, Thangavel R. The parahippocampal gyrus in Alzheimer's disease. Clinical and preclinical neuroanatomical correlates. Ann N Y Acad Sci. 2000;911:254-74.

67. Ulrich JD, Ulland TK, Colonna M, Holtzman DM. Elucidating the role of TREM2 in Alzheimer's disease. Neuron. 2017:94:237-48.

68. Serrano-Pozo A, Frosch MP, Masliah E, Hyman BT. Neuropathological alterations in Alzheimer disease. Cold Spring Harb Perspect Med. 2011;1:a006189.

69. Castellano JM, Kim J, Stewart FR, Jiang H, DeMattos RB, Patterson BW, et al. Human apoE isoforms differentially regulate brain amyloid-beta peptide clearance. Sci Transl Med. 2011;3:89ra57.

70. Kim J, Basak JM, Holtzman DM. The role of apolipoprotein E in Alzheimer's disease. Neuron. 2009;63:287-303.

71. Colonna M. TREMs in the immune system and beyond. Nat Rev Immunol. 2003:3:445-53.

72. Wang Y, Ulland TK, Ulrich JD, Song W, Tzaferis JA, Hole JT, et al. TREM2mediated early microglial response limits diffusion and toxicity of amyloid plaques. J Exp Med. 2016:213:667-75.

73. Leyns CEG, Ulrich JD, Finn MB, Stewart FR, Koscal LJ, Remolina Serrano J, Robinson GO, Anderson E, Colonna M, Holtzman DM. TREM2 deficiency attenuates neuroinflammation and protects against neurodegeneration in a mouse model of tauopathy. Proc Natl Acad Sci. 2017; 114:11524-29.

74. Habib N, Avraham-Davidi I, Basu A, Burks T, Shekhar K, Hofree M, et al. Massively parallel single-nucleus RNA-seq with DroNc-seq. Nat Methods. 2017;14:955-8.

\section{Ready to submit your research? Choose BMC and benefit from:}

- fast, convenient online submission

- thorough peer review by experienced researchers in your field

- rapid publication on acceptance

- support for research data, including large and complex data types

- gold Open Access which fosters wider collaboration and increased citations

- maximum visibility for your research: over $100 \mathrm{M}$ website views per year 